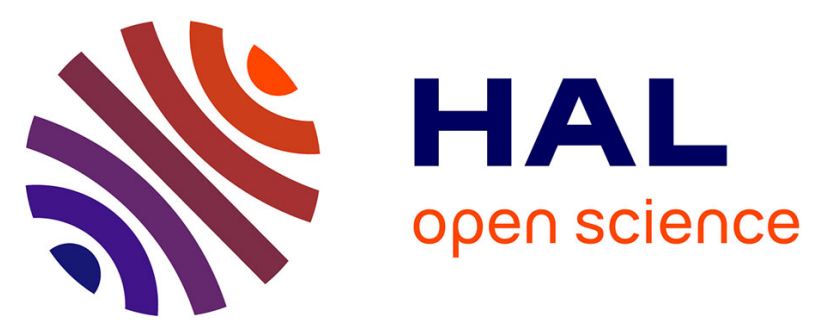

\title{
Rain evaporation, snow melt and entrainment at the heart of water vapor isotopic variations in the tropical troposphere, according to large-eddy simulations and a two-column model
}

\author{
Camille Risi, Caroline Muller, Peter N Blossey
}

\section{To cite this version:}

Camille Risi, Caroline Muller, Peter N Blossey. Rain evaporation, snow melt and entrainment at the heart of water vapor isotopic variations in the tropical troposphere, according to large-eddy simulations and a two-column model. Journal of Advances in Modeling Earth Systems, 2021, 13 (4), 10.1029/2020MS002381 . hal-03025845

\section{HAL Id: hal-03025845 \\ https://hal.science/hal-03025845}

Submitted on 26 Nov 2020

HAL is a multi-disciplinary open access archive for the deposit and dissemination of scientific research documents, whether they are published or not. The documents may come from teaching and research institutions in France or abroad, or from public or private research centers.
L'archive ouverte pluridisciplinaire HAL, est destinée au dépôt et à la diffusion de documents scientifiques de niveau recherche, publiés ou non, émanant des établissements d'enseignement et de recherche français ou étrangers, des laboratoires publics ou privés. 


\section{${ }_{34}$ Contents} vapor.

\title{
Rain evaporation, snow melt and entrainment at the heart of water vapor isotopic variations in the tropical troposphere, according to large-eddy simulations and a two-column model
}

\author{
Camille Risi ${ }^{1}$, Caroline Muller ${ }^{1}$, Peter N. Blossey ${ }^{2}$
}

September 15, 2020

\footnotetext{
${ }^{1}$ Laboratoire de Météorologie Dynamique, IPSL, CNRS, Sorbonne Université, Paris, France

${ }^{2}$ Department of Atmospheric Sciences, University of Washington, Seattle, USA

$(<=140$ characters $)$
}

1. Rain evaporation enriches the tropospheric water vapor more if more snow sublimates before melting and more rain evaporates.

2. Entrainment of dry air reduces the vertical isotopic gradient and limits the tropospheric depletion of water

3. These mechanisms explain the increased depletion of tropospheric water vapor as tropospheric relative humidity increases.

\begin{abstract}
$(<=250$ words $)$

The goal of this study is twofold. First, we aim at developing a simple model as an interpretative framework for the water vapor isotopic variations in the tropical troposphere over the ocean. We use large-eddy simulations to justify the underlying assumptions of this simple model, to constrain its input parameters and to evaluate its results. Second, we aim at interpreting the depletion of the water vapor isotopic composition in the lower and midtroposphere as precipitation increases, which is salient features in tropical oceanic observations. This constitutes a stringent test on the relevance of our interpretative framework. Previous studies, based on observations or models with parameterized convection, have highlighted the roles of deep convective and meso-scale downdrafts, rain evaporation, rain-vapor diffusive exchanges and mixing processes.

The interpretative framework that we develop is a two-column model representing the net ascent in clouds and the subsiding environment. We show that the mechanisms for depleting the troposphere when precipitation rate is larger all stem from the higher tropospheric relative humidity. First, when the relative humidity is larger, less snow sublimates before melting and a smaller fraction of rain evaporates. Both effects lead to more depleted rain evaporation and eventually more depleted water vapor. This mechanism dominates in regimes of large-scale ascent. Second, the entrainment of dry air into clouds reduces the vertical isotopic gradient and limits the tropospheric depletion of water vapor. This mechanism dominates in regimes of large-scale descent.
\end{abstract}

1 Introduction $\quad 2$

1.1 Looking for an interpretative framework for water vapor isotopic profiles . . . . . . . . . . . . 2

1.2 Large-eddy simulation analysis as a guide to design the interpretative framework . . . . . . . . . . 3

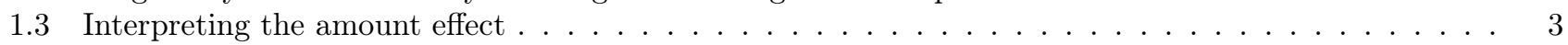


40

41

42

43

44

45

46

47

2 Large-eddy simulations 4

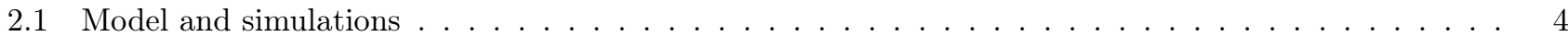

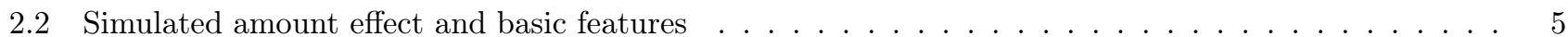

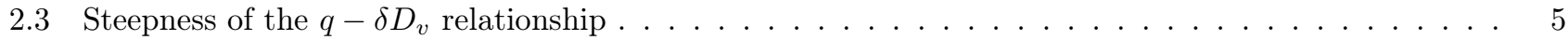

2.4 Effect of de-activating rain-vapor exchanges $\ldots \ldots \ldots \ldots \ldots \ldots \ldots \ldots$

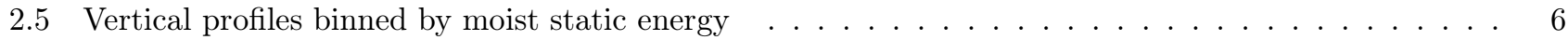

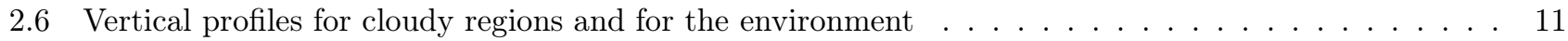

2.7 What controls the isotopic composition of the rain evaporation flux? . . . . . . . . . . . . . 12

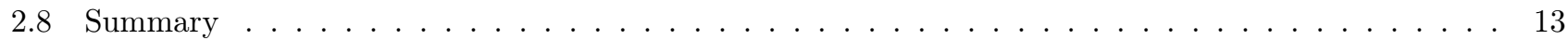

3 A simple two-column model to quantify the relative contributions of different processes 13

3.1 Model equations and numerical application to LES outputs . . . . . . . . . . . . . . . . . 13

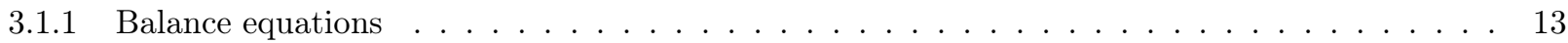

3.1.2 Other simplifying assumptions and differential equations . . . . . . . . . . . . . 15

3.1 .3 Numerical solutions . . . . . . . . . . . . . . . . . . . . . . . . . . . . . 16

3.1 .4 Diagnosed input parameters . . . . . . . . . . . . . . . . . . . . 17

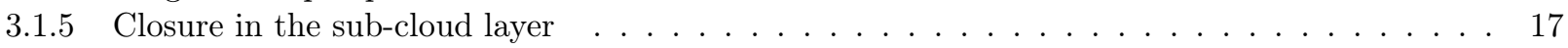

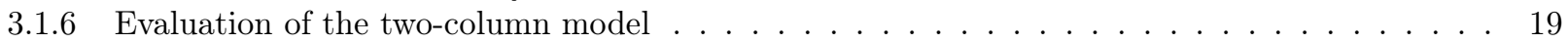

3.2 Decomposition of relative humidity and $\delta D$ variations $\ldots \ldots \ldots \ldots \ldots \ldots \ldots \ldots$

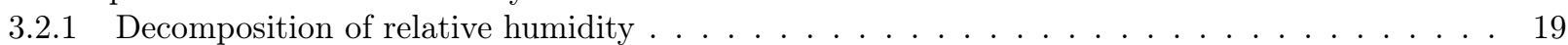

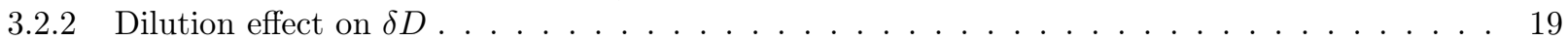

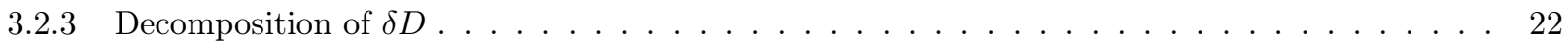

4 Conclusion $\quad 23$

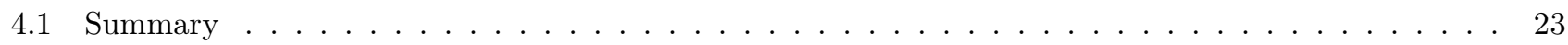

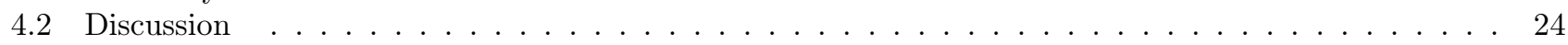

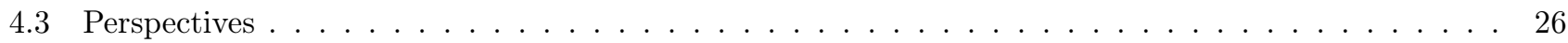

\section{Introduction}

\subsection{Looking for an interpretative framework for water vapor isotopic profiles}

The isotopic composition of water vapor (e.g. its Deuterium content, commonly expressed as $\delta D=\left(R / R_{S M O W}-1\right) \times$ 1000 in \%, where $R$ is the ratio of Deuterium over Hydrogen atoms in the water, and SMOW is the Standard Mean Ocean Water reference) evolves along the water cycle as phase changes are associated with isotopic fractionation. Consequently the isotopic composition of precipitation recorded in paleoclimate archives has significantly contributed to the reconstruction of past hydrological changes ([Wang et al., 2001]). It has also been suggested that observed isotopic composition of water vapor could help better understand atmospheric processes and evaluate their representation in climate models, in particular convective processes ([Schmidt et al., 2005, Bony et al., 2008, Lee et al., 2009, Field et al., 2014]). Yet, water isotopes remain rarely used beyond the isotopic community to answer today's pressing climate questions. A prerequisite to better assess the strengths and weaknesses of the isotopic tool is to better understand what controls spatio-temporal variations in water vapor isotopic composition $\left(\delta D_{v}\right)$ through the tropical troposphere, in particular how convective processes drive these variations.

While there are interpretative frameworks for the controls of free tropospheric humidity ([Sherwood, 1996, Romps, 2014]), no such interpretative framework exist for water isotopes beyond the simple Rayleigh distillation or mixing lines ([Worden et al., 2007, Bailey et al., 2017]). We aim at filling this gap here. The first goal of this paper is thus to design an interpretative framework that could be useful in the future to interpret water vapor isotopic variations in the tropical troposphere in a wide range of contexts. Analogous to that for relative humidity, this framework will also allow us to compare the processes controlling relative humidity and isotopic composition.

Frameworks do exist to interpret the $\delta D_{v}$ in the sub-cloud layer (SCL), such as the [Merlivat and Jouzel, 1979] closure assumption, later extended to account for updrafts and downdrafts ([Risi et al., 2020]). This latter framework highlighted the need to know the steepness of the relationship between $\delta D_{v}$ and humidity $q$ as they evolve with altitude. This motivates us to develop a framework that allows us to predict the $\delta D_{v}$ evolution with altitude in the troposphere. 


\subsection{Large-eddy simulation analysis as a guide to design the interpretative framework}

Many previous studies investigating the processes controlling tropospheric $\delta D_{v}$ have relied on general circulation models that include convective parameterization ([Lee et al., 2007, Bony et al., 2008, Risi et al., 2008, Field et al., 2010]). However, parameterizations include numerous simplifications or assumptions that are responsible for a significant part of biases in the present climate simulated by GCMs and of inter-model spread in climate change projections ([Randall et al., 2003, Stevens and Bony, 2013, Webb et al., 2015]). Here, we thus use large-eddy simulations (LES) as a guide to design the interpretative framework. These high-resolution simulations allows us to explicitly resolve convective motions. These simulations will also provide the input parameters for our interpretative framework, and a benchmark to evaluate its results.

\subsection{Interpreting the amount effect}

In the tropics, it has long been observed that in average over a month or longer, the isotopic composition of the rain is more depleted when the precipitation rate is stronger ([Dansgaard, 1964, Rozanski et al., 1993]). This phenomenon is called the "amount effect". Since most of the precipitation in the tropics is associated with deep convection, understanding the amount effect is a stringent test on our understanding of how convective processes affect the water vapor isotopic composition trough the tropical troposphere. The capacity of our interpretative framework to predict the amount effect will thus be a stringent test on its relevance. The second goal of this study is thus to better understand the processes underlying the amount effect, using the interpretative framework.

[Dansgaard, 1964] hypothesized that the amount effect could be due to the progressive depletion by convective storms of the vapor from which the rain forms, and to rain evaporation and diffusive exchanges between the rain and the vapor. If the case, the amount effect crucially depends on the isotopic composition of the vapor. From a column-integrated water budget perspective, the isotopic composition of precipitation depends on the relative proportion of the precipitation that originates from horizontal advection and from surface evaporation ([Lee et al., 2007, Moore et al., 2014]). More precipitation is generally associated with more large-scale ascent and thus more large-scale convergence. Since vapor from horizontal advection is more depleted than water from surface evaporation because it has already been processed in clouds, the precipitation is more depleted. In this view as well, the amount effect crucially depends on the isotopic composition of the vapor.

Water isotopic measurements in the vapor phase, by satellite or in-situ, have confirmed that increased precipitation was associated with more depleted water vapor ([Worden et al., 2007, Kurita, 2013, Lacour et al., 2017]). Hereafter we will call this the "vapor amount effect". Actually, the precipitation and water vapor isotopic composition often vary in concert ([Kurita, 2013, Tremoy et al., 2014]). In this paper, we will thus focus on understanding the processes underlying the "vapor amount effect".

From previous studies, four hypotheses have emerged to explain the "vapor amount effect":

1. Hypothesis 1: As precipitation rate increases, convective or meso-scale downdrafts bring more depleted vapor from above into the sub-cloud layer (SCL) ([Risi et al., 2008, Kurita et al., 2011, Kurita, 2013]). This is because the water vapor $\delta D\left(\delta D_{v}\right)$ generally decreases with altitude, because as water vapor is lost through condensation and specific humidity $q$ decreases, heavy isotopes are preferentially lost in the condensed phase. This phenomenon is called Rayleigh distillation and is plotted in a $q-\delta D_{v}$ diagram in Figure 1 (blue). However, downdrafts would both decrease $\delta D_{v}$ and $q$. This hypothesis is thus inconsistent with the observation that $q$ generally increases while $\delta D_{v}$ decreases as precipitation rate increases. By itself, this hypothesis cannot be sufficient.

2. Hypothesis 2: As precipitation rate increases, the moistening effect by rain evaporation increases. If rain evaporation is more depleted than the vapor, then it depletes the vapor ([Worden et al., 2007]). The effect of rain evaporation is represented in purple in Figure 1. If the evaporated fraction of the rain is small, rain evaporation acts to deplete the vapor because light isotopes preferentially evaporate.

3. Hypothesis 3: As precipitation rate increases, the rain evaporation is more depleted. For example, if precipitation rate increases, the fraction of rain that evaporates is smaller, which leads the evaporation to be more depleted ([Risi et al., 2008, Risi et al., 2010, Tremoy et al., 2014, Risi et al., 2020], Figure 1, purple). Alternatively, larger precipitation rates typically occur in moister environments, which favors rain-vapor diffusive exchanges rather than pure evaporation [Lawrence et al., 2004, Lee and Fung, 2008]. Since rain comes from higher altitudes, it is more depleted than if in equilibrium with the local vapor, and thus rain-vapor diffusive exchanges favor more depleted evaporation. 

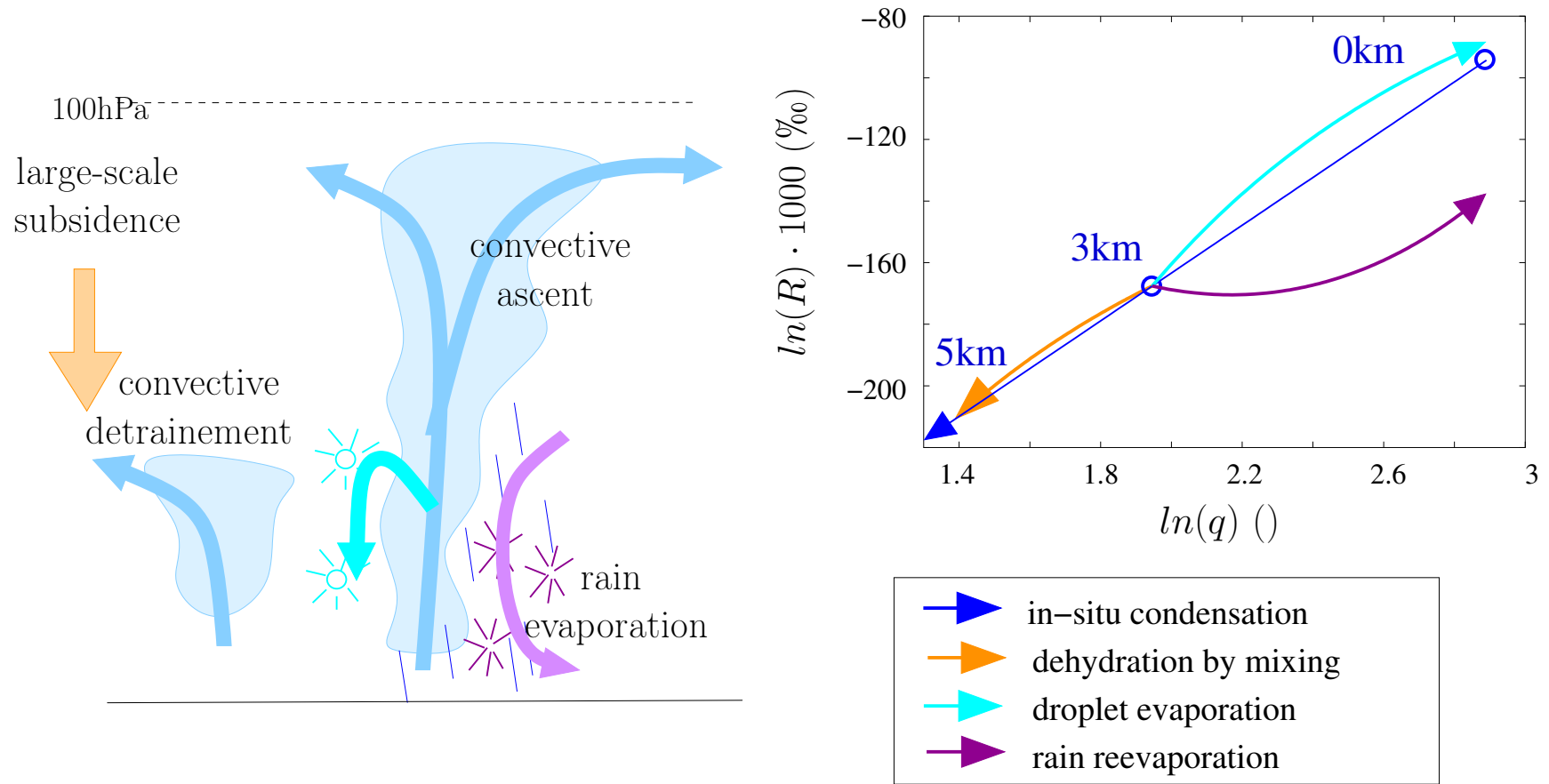

Figure 1: Schematic showing the influence of different processes on $q$ and $\delta D_{v}$. Condensation and immediate loss of condensate in convective updrafts leads to drying and depleting the water vapor following Rayleigh distillation (blue). During evaporation of cloud droplets, each droplet evaporates totally. Since cloud droplets are enriched in heavy isotopes, this moistens the air and enriches the vapor (cyan). In contrast, during evaporation of rain drops, each drop evaporates progressively. Whereas it moistens the air, it depletes the vapor for small evaporation fractions and enriches the vapor for large evaporation fraction (purple). Finally, mixing of subsiding air with air detrained from convective updrafts dehydrates the air and depletes the vapor following a hyperbolic curve, leading to higher $\delta D_{v}$ for a given $q$ compared to Rayleigh (orange). The curves are plotted following simple Rayleigh and mixing lines with approximate values taken from the control LES described later in the article.

4. Hypothesis 4: As precipitation rate increases, dehydration by mixing dominates relatively to dehydration by condensation. Due to the hyperbolic shape of the mixing lines in a $q-\delta D$ diagram, dehydration by mixing with a dry source is associated with a smaller depletion than predicted by Rayleigh distillation [Dessler and Sherwood, 2003, Galewsky and Hurley, 2010, Galewsky and Rabanus, 2016] (Figure 1 orange). [Bailey et al., 2017] argues that in more subsiding regions, mid-tropospheric vapor is more enriched for a given specific humidity because air masses result from the mixing between air subsiding from a higher altitude and shallow convective detrainment.

We notice that hypothesis 2-4 are all associated with an increased steepness as precipitation rate increases (Figure 1 ), consistent with the key role of the steepness of the $q-\delta D_{v}$ relationship in depleting the SCL water vapor highlighted by [Risi et al., 2020]. The mechanisms underlying these hypotheses will thus have to be key ingredients of our interpretative framework.

The LES will be described and analyzed in section 2. The interpretative framework will be designed and used to interpret the "vapor amount effect" in section 3. Finally, section 4 will offer a summary, some discussion and perspectives.

\section{Large-eddy simulations}

\subsection{Model and simulations}

We use the same LES model as in [Risi et al., 2020], namely the System for Atmospheric Modeling (SAM) nonhydrostatic model ([Khairoutdinov and Randall, 2003]), version 6.10.9, which is enabled with water isotopes ([Blossey et al., 2010 This model solves anelastic conservation equations for momentum, mass, energy and water, which is present in the 
model under six phases: water vapor, cloud liquid, cloud ice, precipitating liquid, precipitating snow, and precipitating graupel. We use the bulk, mixed-phase microphysical parameterization from [Thompson et al., 2008] in which water isotopes were implemented ([Moore et al., 2016]).

The control simulation ("ctrl") is three-dimensional, with a doubly-periodic domain of $96 \mathrm{~km} \times 96 \mathrm{~km}$. The horizontal resolution is $750 \mathrm{~m}$. There are 96 vertical levels. The simulation is run in radiative-convective equilibrium over an ocean surface. The sea surface temperature (SST) is $30^{\circ} \mathrm{C}$. There is no rotation and no diurnal cycle. In this simulation, there is no large-scale circulation.

The amount effect can be seen only if the precipitation increase is associated with a change in the large-scale circulation ([Bony et al., 2008, Dee et al., 2018, Risi et al., 2020]). To compare ctrl to simulations with larger and smaller precipitation rate, we thus run simulations with a large-scale vertical velocity profile, $\omega_{L S}$. This profile is used to compute large-scale tendencies in temperature, humidity and water vapor isotopic composition. We compute large-scale vertical advection by a simple upstream scheme [Godunov, 1959]. In the computation, large-scale horizontal gradients in temperature, humidity and isotopic composition are neglected, i.e. there are no large-scale horizontal advective forcing terms. The large-scale vertical velocity $\omega_{L S}$ has a cubic shape so as to reach its maximum $\omega_{L S \max }$ at a pressure $p_{\max }=500 \mathrm{hPa}$ and to smoothly reach 0 at the surface and at $100 \mathrm{hPa}$ [Bony et al., 2008]. We analyze here simulations with $\omega_{L S \max }=-60 \mathrm{hPa} / \mathrm{d}$ (" $\omega_{L S}-60$ "), corresponding to typical deep convective conditions in the inter-tropical convergence zone, and $\omega_{L S \max }=+20 \mathrm{hPa} / \mathrm{d}$ (" $\omega_{L S}+20$ "), corresponding to subsiding trade-wind conditions.

The simulations are run for 50 days and the last 10 days are analyzed. We use instantaneous outputs that are generated at the end of each simulation day.

\subsection{Simulated amount effect and basic features}

Figure 2a shows that the ctrl, $\omega_{L S}-60$ and $\omega_{L S}+20$ simulations allow us to capture the amount effect both in the vapor and in the precipitation, which vary in concert. In case of large-scale ascent, the domain-mean relative humidity is larger than in ctrl by more than $10 \%$ (Figure $2 \mathrm{~b}$ ), while $\delta D_{v}$ is more depleted by more than $50 \%$, in most of the troposphere (Figure 2c). We can see that the $\delta D_{v}$ difference at all altitudes is similar to that in the SCL. This confirms that understanding what controls the SCL $\delta D_{v}$ is key to understand what controls $\delta D_{v}$ at all altitudes ([Risi et al., 2020]). This also explains why models that assume constant SCL $\delta D_{v}$ show very little sensitivity to all kinds of convective and microphysical processes ([Duan et al., 2018]). We can also see that Rayleigh distillation alone (dashed line) is a poor predictor of $\delta D_{v}$ profiles and of their sensitivity to large-scale ascent.

\subsection{Steepness of the $q-\delta D_{v}$ relationship}

With the goal of understanding the amount effect, as a first step [Risi et al., 2020] focused on understanding what controls the $\delta D_{v}$ in the SCL, because the SCL ultimately feeds the water vapor at all altitudes in the troposphere. They identified the key role of the steepness of the $q-\delta D_{v}$ relationship of vertical profiles in the lower troposphere. This steepness determines the efficiency with which updrafts and downdrafts near the SCL top deplete the SCL. To understand what controls $\delta D_{v}$ in the SCL and thus everywhere in the troposphere, we thus need to understand what controls the steepness of the $q-\delta D_{v}$ relationship.

The vertical profiles of $\ln (R)$ as a function of $\ln (q)$ for each simulation show a nearly linear relationship (Figure 2d), consistent with a Rayleigh-like distillation process (Figure 1). If the vertical profiles were dominated by mixing processes, as in hypothesis 4, the relationship would look convex ([Bailey et al., 2017], Figure 1 orange). Rather, in $\omega_{L S}-60$, the curve looks concave near the melting level, consistent with an effect of rain evaporation (Figure 1 purple).

To better quantify the steepness of the $q-\delta D_{v}$ relationship, we define the $q-\delta D_{v}$ steepness $\alpha_{z}$, as the effective fractionation coefficient that would be needed in a distillation to fit the simulated joint $q-\delta D$ evolution ([Risi et al., 2020]):

$$
\alpha_{z}=1+\frac{\ln (R(z) / R(z-d z))}{\ln (q(z) / d(z-d z))}
$$

The steepness $\alpha_{z}$ in the ctrl simulation is smaller than that predicted by Rayleigh distillation, i.e. $\alpha_{z}<$ $\alpha_{e q}$, especially at higher altitudes (Figure 2e) (section 3.2.2 will demonstrate that it is due to entrainment). In case of large-scale ascent, just above the SCL top, $\alpha_{z}-1$ is more than three times larger in $\omega_{L S}-60$ than in ctrl. The increased steepness leads the updrafts and downdrafts to deplete more efficiently the SCL water vapor ([Risi et al., 2020]), and eventually the full tropospheric profile. Conversely, in $\omega_{L S}+20$, the steepness is smaller and 
responsible for more enriched SCL. Our interpretative framework will allow us to interpret these features (section $3)$.

\subsection{Effect of de-activating rain-vapor exchanges}

According to hypotheses 2 and 3, the isotopic composition of the rain plays a key role in the "vapor amount effect". At a given instant and for a small increment of rain evaporation fraction, the isotopic composition of the evaporation flux $R_{e v}$ is simulated following [Craig and Gordon, 1965]:

$$
R_{e v}=\frac{R_{r} / \alpha_{e q}-h_{e v} \cdot R_{v}}{\alpha_{K} \cdot\left(1-h_{e v}\right)}
$$

where $R_{r}$ and $R_{v}$ are the isotopic ratios in the liquid water and water vapor, $\alpha_{e q}$ and $\alpha_{K}$ are the equilibrium and kinetic fractionation coefficient and $h_{e v}$ is the relative humidity. In order to test hypotheses 2 and 3 , we run additional simulations similar to ctrl and $\omega_{L S}-60$ but without any fractionation during rain evaporation, named "nofrac", where $R_{e v}=R_{r}$. We also run additional simulations with fractionation during evaporation, but with rain-vapor diffusive exchanges de-activated, named "nodiff", where $R_{e v}=R_{r} / \alpha_{e q} / \alpha_{K}$.

When fractionation during rain evaporation is de-activated, $\delta D_{v}$ is more enriched, consistent with a more enriched composition of rain evaporation (Figure 3a). In addition, the $\delta D_{v}$ difference between $\omega_{L S}-60$ and ctrl is reduced by about 70\% compared to when all isotopic exchanges are considered (Figure 3c, red). This confirms that fractionation during rain evaporation plays a key role in the "vapor amount effect". When rain-vapor diffusive exchanges are de-activated, the $\delta D_{v}$ difference between $\omega_{L S}-60$ and $\operatorname{ctrl}$ is reduced by about $30 \%$ compared to when all isotopic exchanges are considered (Figure 3c, green). Rain-vapor vapor diffusive exchanges thus play an important role as well.

We note that the $\delta D_{v}$ difference between the simulations is remarkably constant with altitude (Figure 3a,c), although we expect strong vertical variations in rain evaporation. This is consistent with the important role of the SCL $\delta D_{v}$ as an initial condition for the full $\delta D_{v}$ profile. We also note that more enriched $\delta D_{v}$ profiles are associated with a reduced lower-tropospheric steepness $\alpha_{z}$ just above the SCL, and larger $\delta D_{v}$ differences between simulations are associated with larger differences in lower-tropospheric $\alpha_{z}$. This is consistent with the SCL $\delta D_{v}$ being mainly driven by the steepness $\alpha_{z}$ just above the SCL ([Risi et al., 2020]). Finally, the reduced "vapor amount effect" in "nofrac" leads to a reduced amount effect in the precipitation $\delta D$ as well (Figure 3c, circles). This shows that the column-integrated water budget ([Lee et al., 2007, Moore et al., 2014]) cannot by itself predict the amount effect, since it depends on the isotopic composition of the advected vapor, which can greatly vary depending on the detalied representation of rain evaporation processes.

To summarize, in the total $\delta D_{v}$ difference between $\omega_{L S}-60$ and ctrl, there is about one third due to fractionation during evaporation, one third due to rain-vapor diffusive exchanges, and one third that would remain even in absence of any fractionation during evaporation. These tests suggest that hypotheses 2 and/or 3 play a key role in the "vapor amount effect". In the next sections, we aim at better understanding how rain evaporation impacts $\delta D_{v}$ profiles.

\subsection{Vertical profiles binned by moist static energy}

Previous studies have shown that analyzing variables in isentropic coordinates was a powerful tool to categorize the different convective structures: undiluted updrafts, diluted updrafts, saturated and unsaturated downdrafts, and the environment ([Kuang and Bretherton, 2006, Pauluis and Mrowiec, 2013]). This method also has the advantage of filtering out gravity waves. It has been applied to the analysis of a wide range of convective systems ([Mrowiec et al., 2015, Mrowiec et al., 2016, Dauhut et al., 2017, Chen et al., 2018]).

Here we use the frozen moist static energy $m$ as a conserved variable because it is conserved during condensation and evaporation of both liquid and ice water ([Muller and Romps, 2018, Hohenegger and Bretherton, 2011]).

$$
m=c_{p d} \cdot T+g \cdot z+L_{v} \cdot q_{v}-L_{f} \cdot q_{i}
$$

where $c_{p d}$ is the specific heat of dry air, $T$ is temperature, $g$ is gravity, $z$ is altitude, $L_{v}$ and $L_{f}$ are the latent heat of vaporization and fusion, and $q_{i}$ is the total ice water content (cloud ice, graupel and snow). At each level, we categorize all grid points into bins of $m$ with a width of $0.4 \mathrm{~kJ} / \mathrm{kg}$.

The domain-mean frozen moist static energy $m$ decreases from the upper troposphere down to about $5 \mathrm{~km}$, due to the loss of energy by radiative cooling, and then increases down to the surface due to the input of energy by surface fluxes (Figure 4, solid black line). Based on this diagram, we can identify four kinds of air parcels: 
(a)

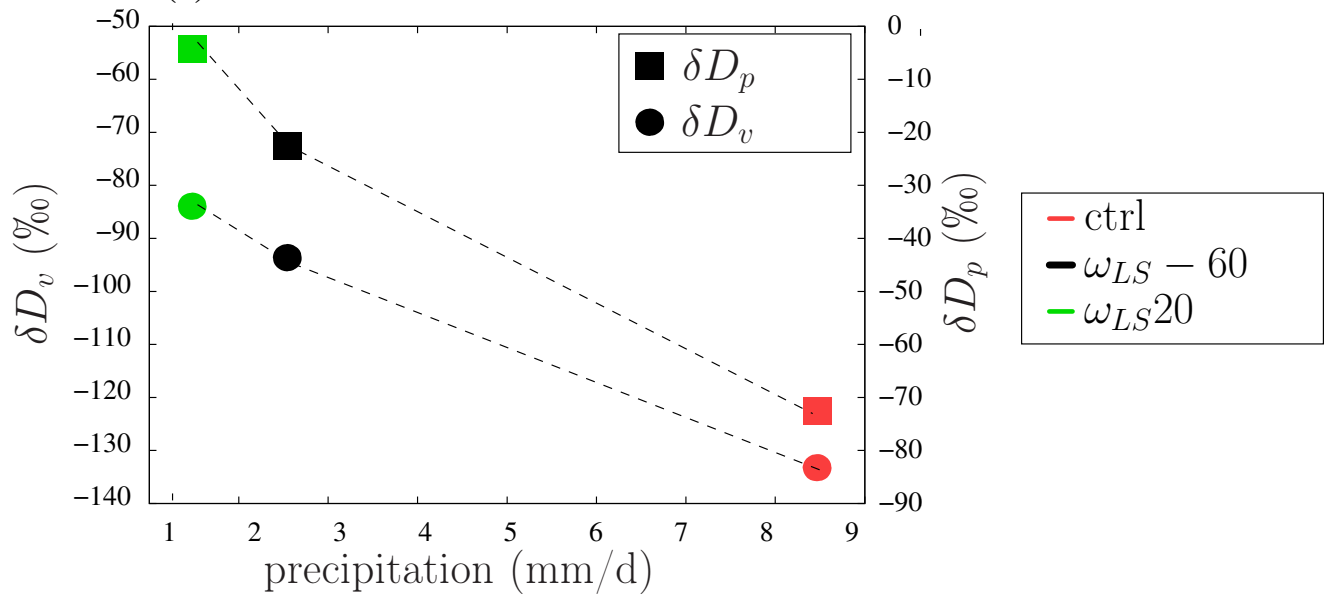

(b)

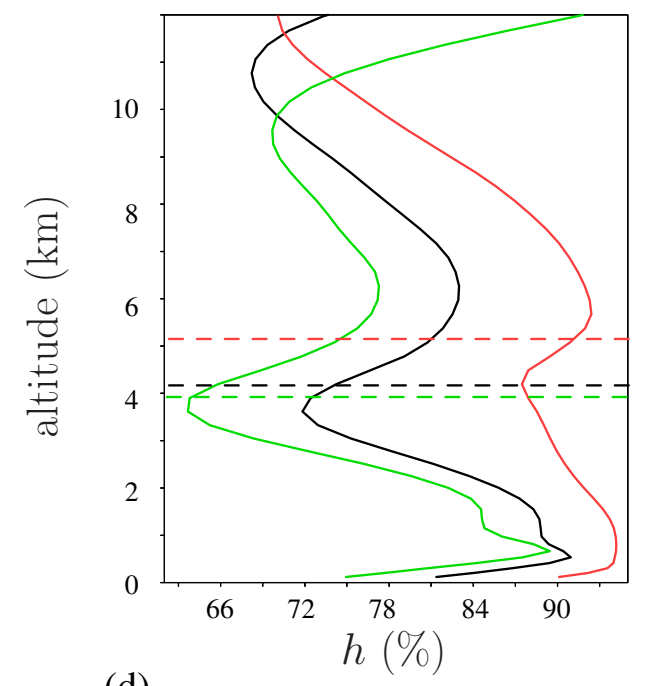

(d)

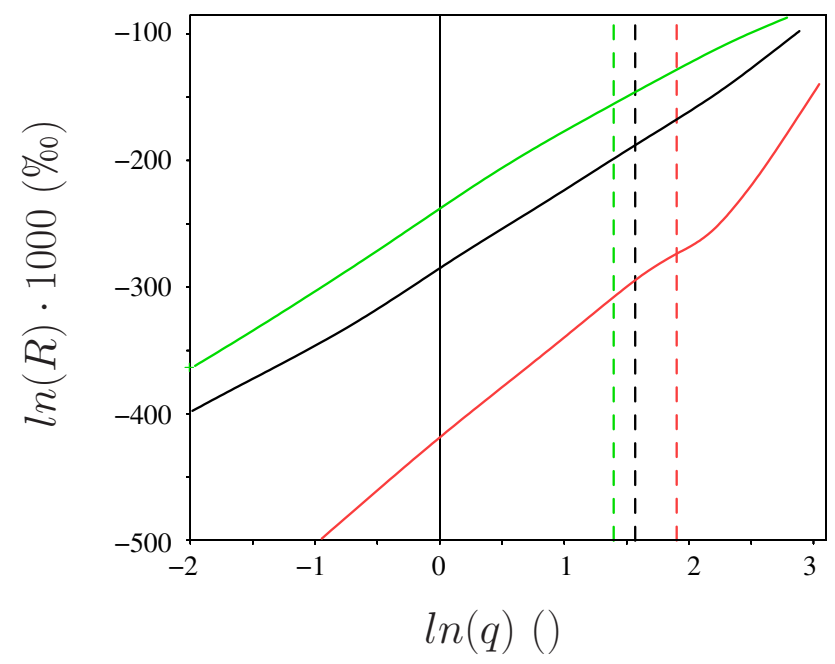

(c)
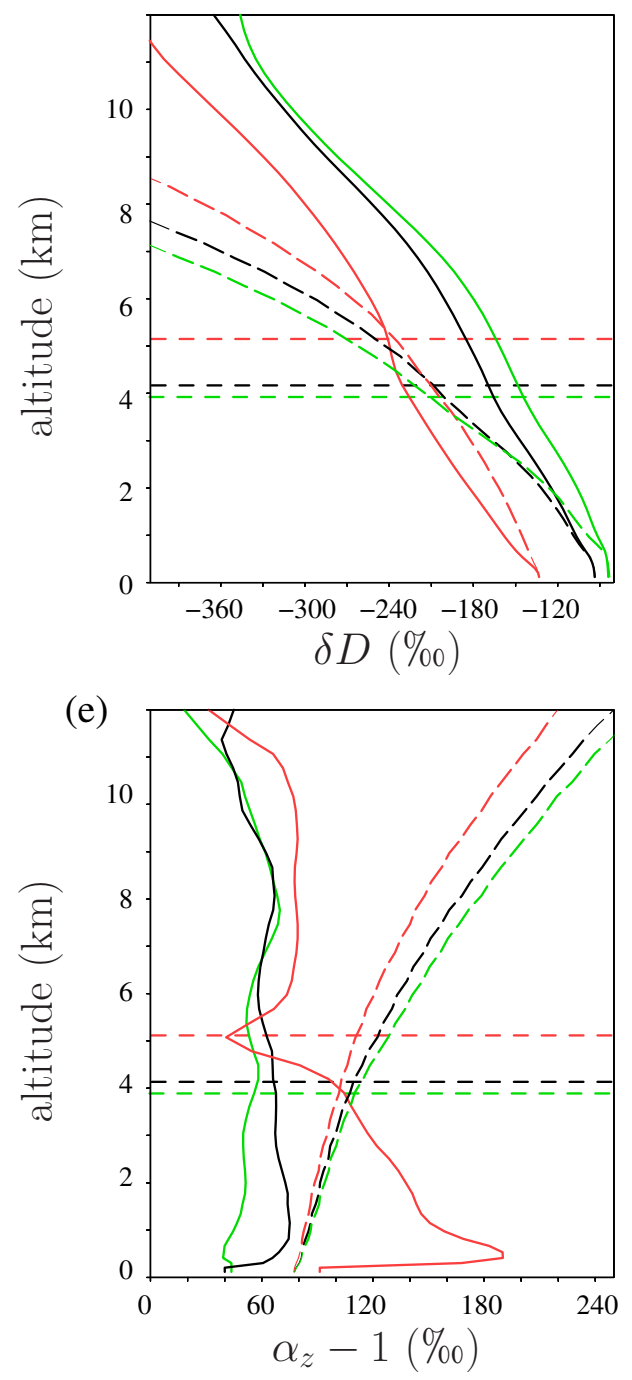

Figure 2: (a) Water vapor (circles) and precipitation (squares) $\delta D_{v}$ as a function of precipitation. Vertical distribution of relative humidity (b), water vapor $\delta D$ (c) and $\alpha_{z}$ (e) in ctrl (black), $\omega_{L S}-60$ (red) and $\omega_{L S}+20$ (green). (c) $\ln (R(z)) \cdot 1000$ as a function of $\ln (q(z))$ for different altitudes. In $\mathrm{c}$ and e, dashed lines indicate the prediction by Rayleigh distillation. The horizontal lines show the altitude of the melting level. 
(a)
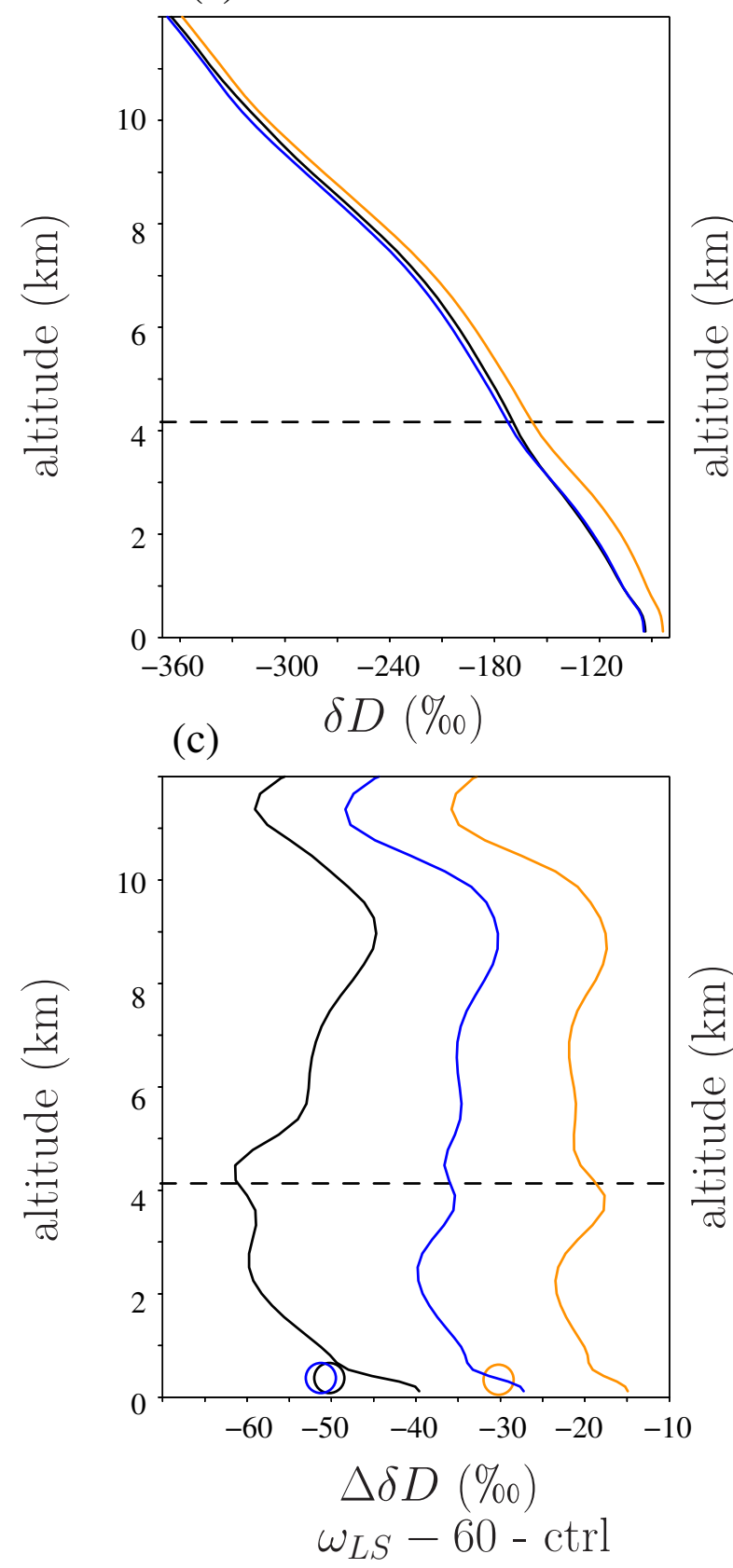

(b)

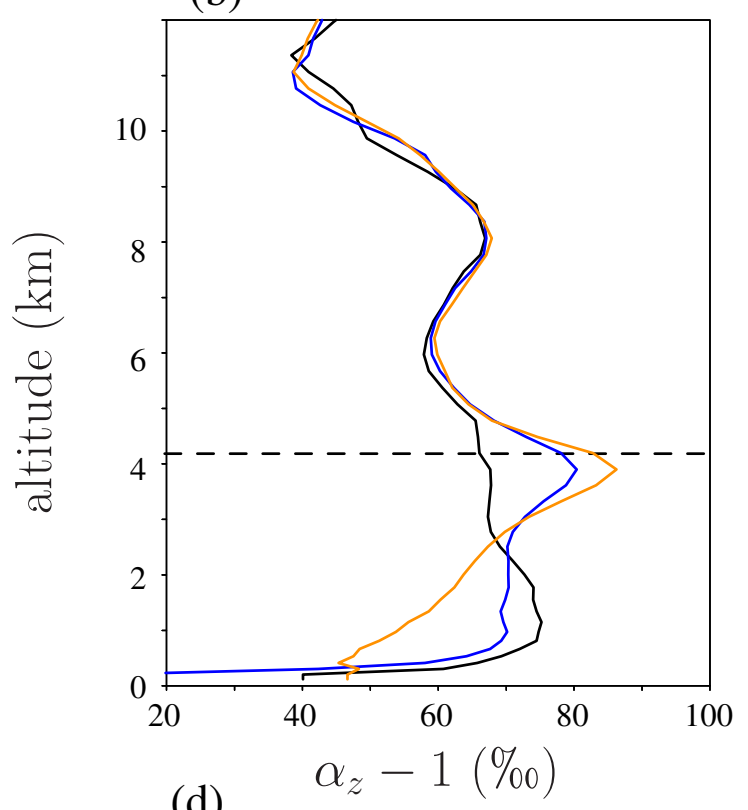

(d)

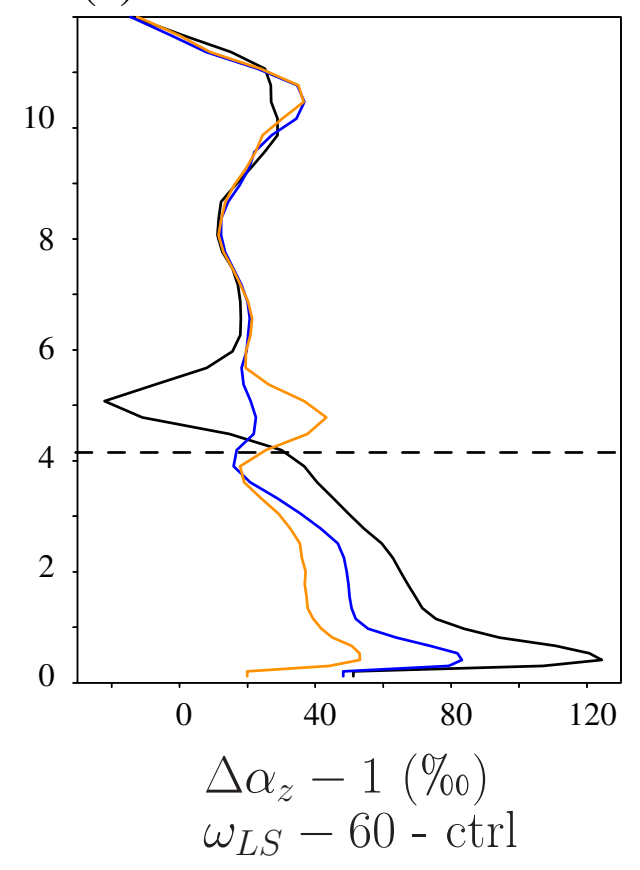

$$
\begin{aligned}
& \text { - ctrl } \\
& \text { - nofrac } \\
& \text { - nodiff }
\end{aligned}
$$

Figure 3: (a) Vertical distribution of $\delta D_{v}$ for ctrl, when fractionation during liquid evaporation is turned on (black) or off (red) and when liquid-vapor equilibration is turned off (green).

(b) Same as (a) for the vertical profiles of $\alpha_{z}$.

(c) $\delta D_{v}$ difference between the $\omega_{L S}-60$ and ctrl, with (black) and without (red) fractionation during evaporation and when liquid-vapor equilibration is turned off (green). The circles illustrate the difference in the precipitation $\delta D$.

(d) Same as (c) but for $\alpha_{z}$. 
(a)

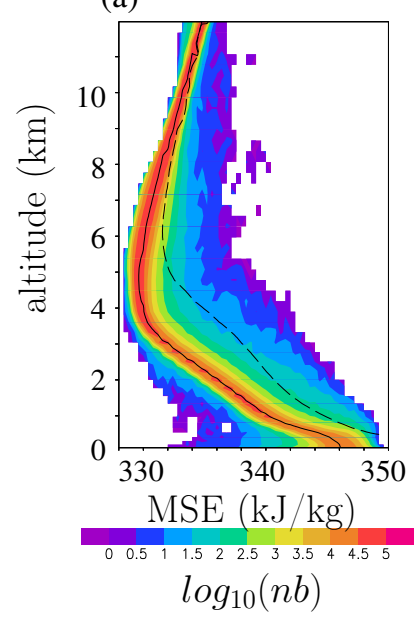

(e)

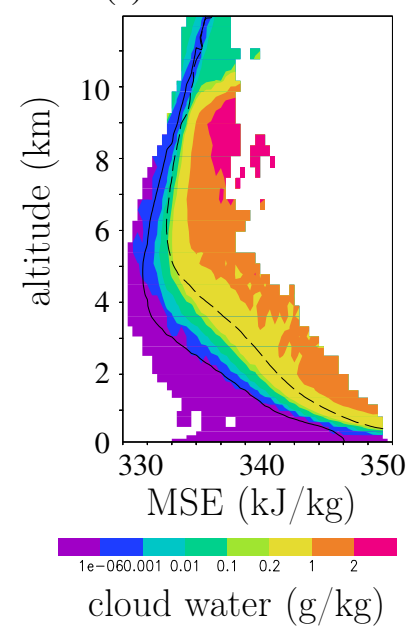

(b)

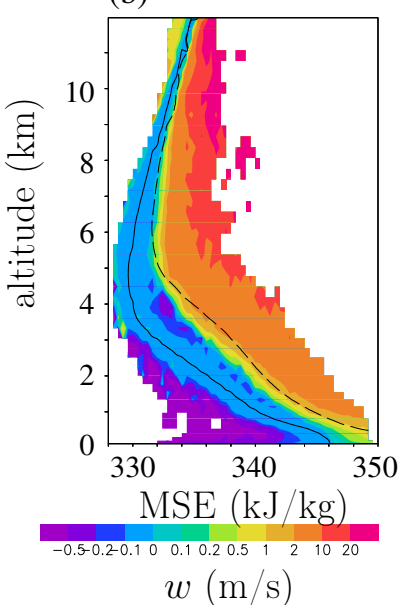

(f)

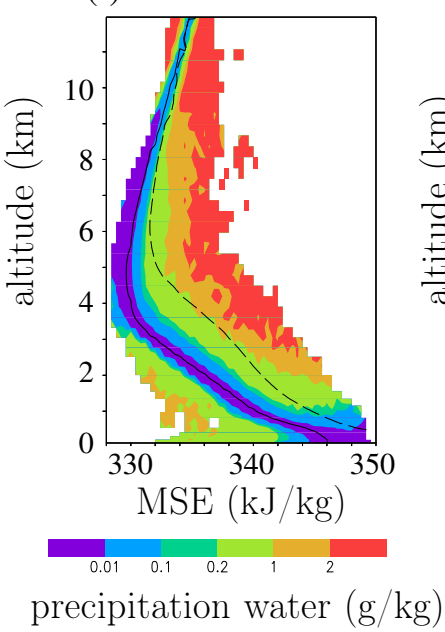

(c)

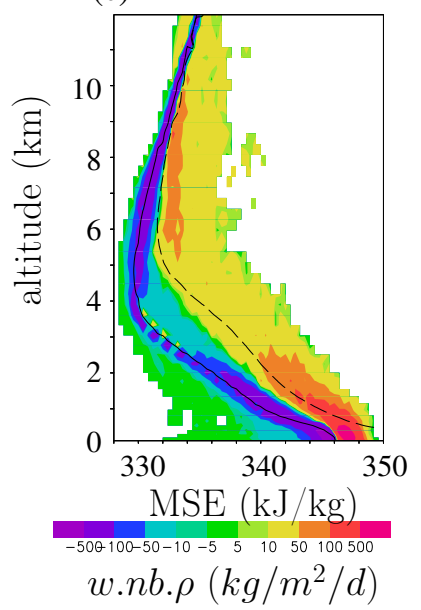

(g)

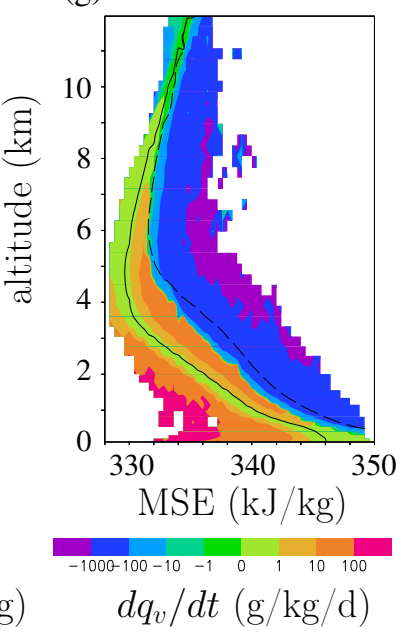

(d)

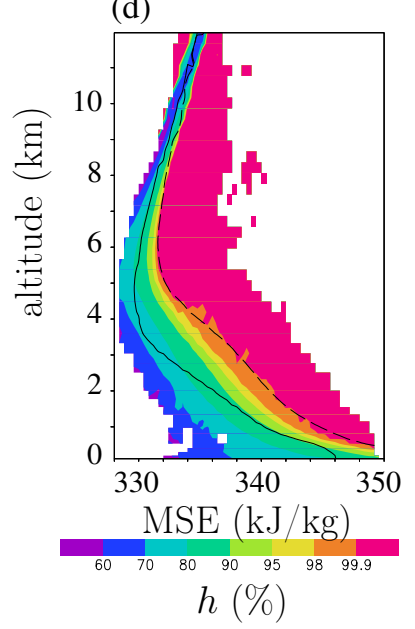

(h)

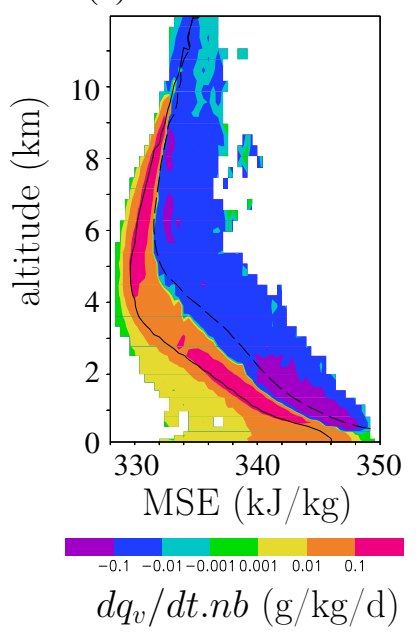

Figure 4: Variables binned as a function of frozen moist static energy $m$ and of altitude, for the ctrl simulation: (a) number of samples, (b) vertical velocity anomaly, (c) vertical mass flux (vertical velocity multiplied by the proportion of samples and density), (d) relative humidity, (e) cloud water content mixing ratio (liquid and ice), (f) precipitating water mixing ratio (rain, graupel and snow), (g) evaporation and condensation tendency (positive in case of evaporation, negative in case of condensation), (h) $\delta D_{v}$ anomaly, (i) $(\phi-1) \cdot 1000$, where $\phi=R_{e v} / R_{v}$; it is expressed in \%. The solid black line show the domain-mean frozen moist static energy, while the dashed black line shows the frozen moist static energy at saturation. 

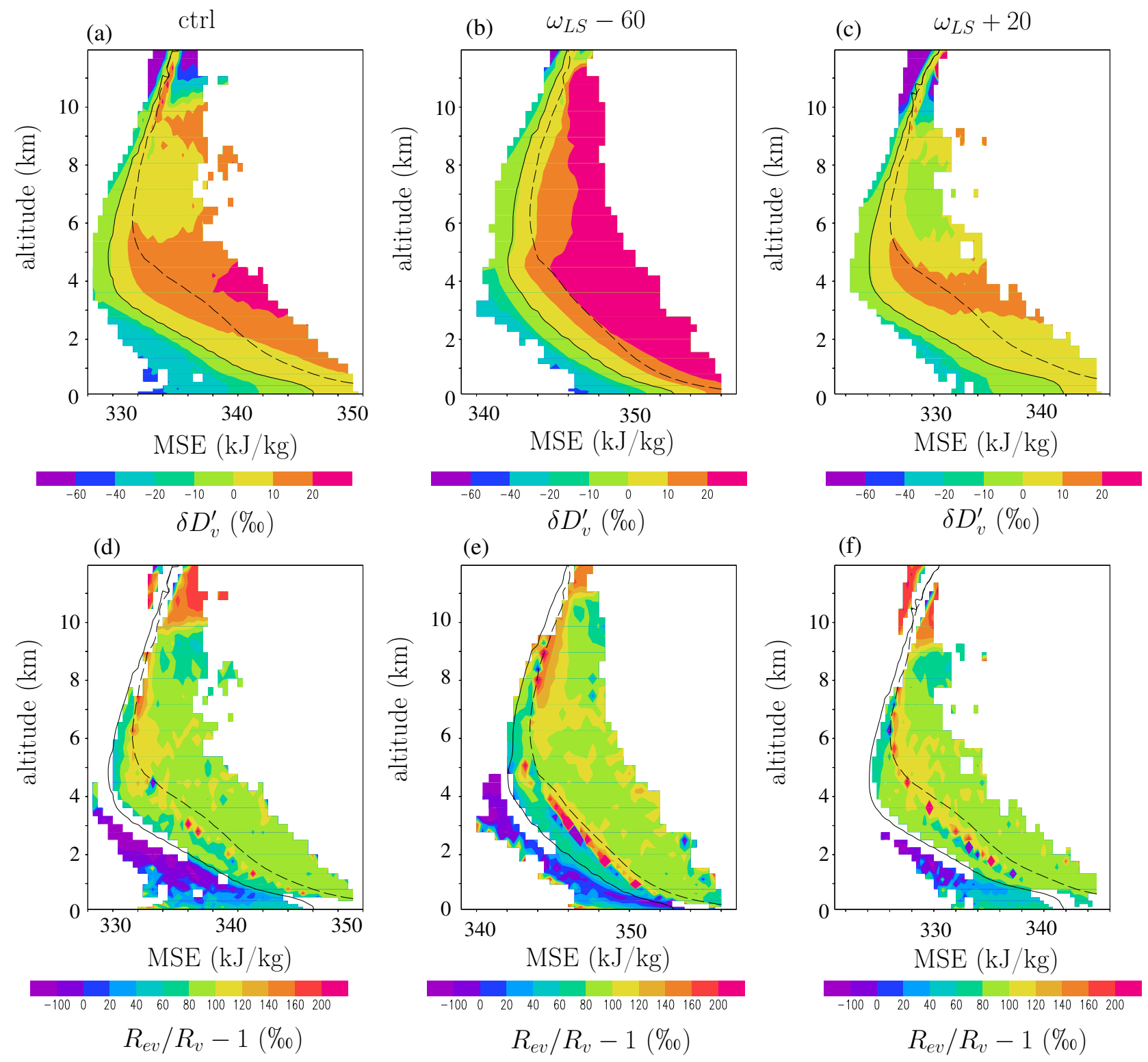

Figure 5: (a,d) As for Figure 4 but for (a) $\delta D_{v}$ anomaly, (d) $(\phi-1) \cdot 1000$, where $\phi=R_{e v} / R_{v}$; it is expressed in \%. (b,e) As for $(\mathrm{a}, \mathrm{d})$ but for $\omega_{L S}-60$. (c,f) As for $(\mathrm{a}, \mathrm{d})$ but for $\omega_{L S}+20$. 
1. Environment. They correspond to air parcels whose $m$ is close to the domain-mean (solid black). They are the most numerous (Figure 4a). Their vertical velocity is slightly descending (Figure 4b), but because they are very numerous, they account for most of the downward mass flux (Figure 4c). Their relative humidity is close to the domain-mean (Figure 4d), they contain only a small cloud water and rain content and phase changes are very slow (Figure 4e-g). However, because they cover most of the domain, they contribute significantly to the evaporation in the domain-mean (Figure $4 \mathrm{~h}$ ).

2. Cloudy updrafts. They correspond to air parcels on the right of the domain-mean $m$ and whose bin-mean vertical velocity is ascending (Figure $4 \mathrm{~b}$ ). If air rose adiabatically from the SCL, they would conserve their $m$ and they would be located completely on the right of the diagram. In practice, $m$ decrease because the environment air is progressively entrained into ascending parcels. In the diagrams, parcels are more diluted when they are closed to the domain-mean, and less diluted when they are more to the right. In spite of their dilution with the environment, their humidity is at saturation ( (Figure 4d). They contain a lot of cloud and precipitating water, and vapor undergoes condensation (Figure 4e-g).

3. Cloudy downdrafts. They correspond to air parcels on the right of the domain-mean $m$ and whose bin-mean vertical velocity is descending (Figure $4 \mathrm{~b}$ ). They are more diluted than cloudy updrafts. Their humidity is below saturation (Figure 4d). They contain cloud and precipitating water, but these hydrometeor undergo evaporation (Figure 4e-g). Located around the cloudy updrafts in the real space, they mainly correspond to subsiding shells ([Glenn and Krueger, 2014]).

4. Precipitating downdrafts. They correspond air parcels on the bottom-left of the diagrams, with lower $m$ relative to the domain-mean. They are among the most strongly descending air parcels (Figure 4b), but since they are scarce (Figure 4b), they contribute little to the total descending mass flux (Figure 4c). They are very dry, with no cloud water, but with precipitating water (Figure 4d-f). We interpret these parcels as unsaturated, precipitating downdrafts. Strong evaporation of rain occur in these downdrafts (Figure $4 \mathrm{~g}$ ), but because they cover only a small fraction of the domain, they contribute little to the evaporation in the domain-mean (Figure 4h).

The isotopic composition of water vapor is the strongest in the least diluted updrafts, and the most depleted in the precipitating downdrafts (Figure 5a). To assess the effect of phase changes, we plot $\phi=R_{e v} / R_{v}$, where $R_{e v}$ is the ratio of the water vapor tendency associated with phase changes (evaporation in downdrafts and in the environment, or condensation in cloudy updrafts) and $R_{v}$ is the isotopic ratio of the water vapor. In cloudy updrafts, $\phi-1$ is about $100 \%$ in the lower troposphere and increases with height (Figure 5b). This corresponds roughly to equilibrium fractionation during condensation. In cloudy downdrafts, $\phi-1$ is also about $100 \%$. This means that cloud droplets evaporate totally without fractionation. In contrast, in precipitating downdrafts, $\phi-1$ is much lower. It is around $30 \%$ below $1 \mathrm{~km}$. The fact that $\phi-1$ is positive is consistent with the fact that rain evaporation in the SCL acts to slightly enrich the water vapor ([Risi et al., 2020]). In contrast, between 2 and $3 \mathrm{~km}, \phi-1$ is around $-100 \%$ : at these levels, rain evaporation acts to deplete the water vapor, consistent with [Worden et al., 2007].

These diagrams look qualitatively similar for the other simulations. One noticeable difference is that in $\omega_{L S}-60$, the $\delta D_{v}$ contrast between the environment and the cloudy regions is larger (Figure $5 \mathrm{~b}$ ). This may be associated with the more depleted evaporation of the rain in precipitating downdrafts, and even of cloud droplets in cloudy downdrafts (Figure 5e). Conversely, in $\omega_{L S}+20$, the $\delta D_{v}$ contrast between the environment and the cloudy regions is larger (Figure 5c). To quantitatively compare the different simulations, now we plot vertical profiles of variables in average over cloudy regions and over the environment.

\subsection{Vertical profiles for cloudy regions and for the environment}

Here we chose to define cloudy regions as all parcels with a cloud (liquid or ice) water content greater than $10^{-6} \mathrm{~g} / \mathrm{kg}$ (e.g. [Thayer-Calder and Randall, 2015]). In this loose definition, "cloudy regions" correspond to both cloudy updrafts and downdrafts, while the "environment" includes both the environment and precipitating downdrafts. Including the cloudy downdrafts into the cloudy regions is justified by the fact that a significant portion of the water condensed in cloudy updrafts subsequently evaporate in these cloudy downdrafts, without directly affecting the environment. Our results below are not crucially sensitive to the definition of the distinction between cloudy regions and the environment, provided that the definition of cloudy regions is not too restrictive (Text S1).

Cloudy regions cover only a few percent of the domain (Figure 6a). The fraction of water condensed in cloudy regions that evaporates into the environment, estimated as $f_{e v}=-(d q / d t)_{\text {env }} /(d q / d t)_{c l o u d}$, where $(d q / d t)_{\text {env }}$ and $(d q / d t)_{c l o u d}$ are the humidity tendencies associated with phase changes in average in the environment and in the 
(a)

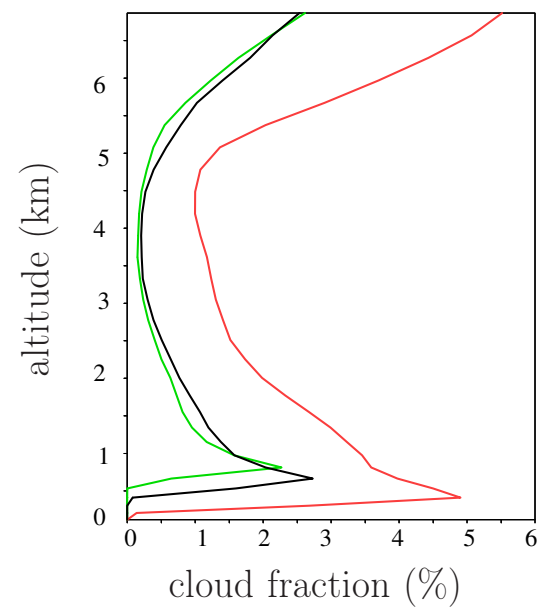

(b)

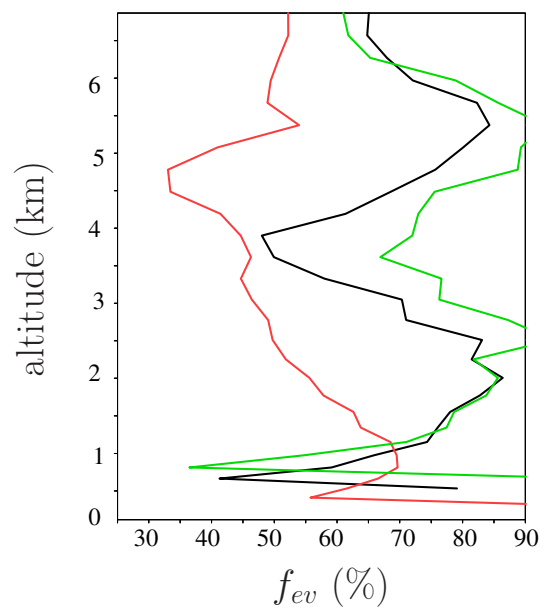

(c)

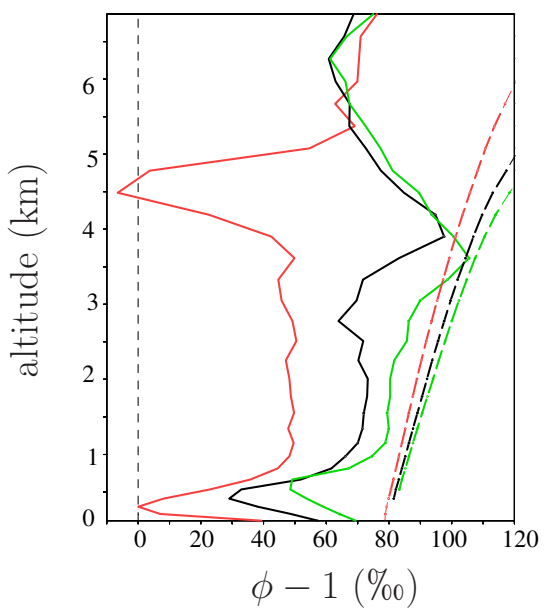

$$
\begin{aligned}
& -\operatorname{ctrl} \\
& -\omega_{L S}-60 \\
& -\omega_{L S}+20
\end{aligned}
$$

Figure 6: (a) fraction of the domain-area covered by cloudy regions. (b) Fraction of the water condensed in cloudy regions that evaporates into the environment, $f_{e v}$. (c) $(\phi-1) \cdot 1000$ (solid) and $\left(\alpha_{e q}-1\right) \cdot 1000$ (dashed), where $\phi=R_{e v} / R_{e}$ and $\alpha_{e q}$ is the equilibrium fractionation coefficient. Both are expressed in \%. The black, red and green lines are for ctr, $\omega_{L S}-60$ and $\omega_{L S}+20$ respectively.

cloudy region respectively, varies between $30 \%$ and $90 \%$, depending on altitude (Figure $6 \mathrm{~b}$ ). It is smaller in $\omega_{L S}-60$ and than in ctrl, because the environment is moister.

Figure 6c plots $\phi=R_{e v} / R_{e}$, where $R_{e v}=\left(d q_{H D O} / d t\right)_{e n v} /(d q / d t)_{e n v},\left(d q_{H D O} / d t\right)_{e n v}$ is the HDO tendency associated with phase changes in the environment and $R_{e}$ is the isotopic ratio in the environment. In ctrl,$\phi>1$ : the evaporation has an enriching effect on the environment. Yet, $\phi<\alpha_{e q}$ : the evaporation is not as enriching as if there was total evaporation of condensate. In $\omega_{L S}-60, \phi$ is smaller than in ctrl: rain evaporation has a weaker enriching effect. At $4.5 \mathrm{~km}$, near the melting level, there is a small layer where $\phi<1$ : at this level, the rain evaporation has a depleting effect on the water vapor. The overall enriching effect of evaporation contradicts hypothesis 2 , but the weaker enriching effect in $\omega_{L S}-60$ than in ctrl supports hypothesis 3 . Conversely, $\phi$ is larger in $\omega_{L S}+20$.

\subsection{What controls the isotopic composition of the rain evaporation flux?}

Why is $\phi$ smaller in $\omega_{L S}-60$ and higher in $\omega_{L S}+20$ than in ctrl? It could be because rain-vapor exchanges in a moister environment leads the evaporation to have a more depleting effect ([Lawrence et al., 2004, Risi et al., 2008]), or because rain evaporation is more depleted when the evaporated fraction is small ([Risi et al., 2008, Tremoy et al., 2014]), or because the rain itself is more depleted. We aim here at quantifying these different effects.

Figure 7 a plots the vertical profiles of rain $\delta D$ (solid). Below the melting level, the rain is very close to isotopic equilibrium with the vapor (dashed). Above the melting level, the rain is more enriched than if in equilibrium due to rain lofting. Near the melting level for simulation $\omega_{L S}-60$, the rain is anomalously depleted. This is due to snow melt. Since the snow forms higher in altitude, it is more depleted than the rain. It thus imprints its depleted signature on the rain when melting. In $\omega_{L S}-60$, the moist middle troposphere prevents most of the snow from sublimating: $24 \%$ of the precipitation is made of snow at the melting level. The rain is thus strongly depleted by snow melt. In contrast, in ctrl and $\omega_{L S}+20$, the drier middle troposphere favors snow sublimation: only $8 \%$ and $3 \%$ of the precipitation is made of snow at the melting level respectively.

The quick equilibration between the rain and vapor motivates us to use a simple equation in which some mass $q_{l 0}$ of rain, with isotopic ratio $R_{l 0}$, partially evaporates and isotopically equilibrates with some mass $q_{v 0}$ of vapor, with isotopic ratio $R_{v 0}$. As explained in text $\mathrm{S} 2$, if $q_{l 0} \gg q_{v 0}$, we get: 


$$
\phi=\frac{\lambda}{\alpha_{e q}-f_{e v} \cdot\left(\alpha_{e q}-1\right)}
$$

where $\phi=R_{e v} / R_{v 0}, \lambda=R_{l 0} / R_{v 0}, R_{e v}$ is the isotopic ratio of the rain evaporation flux, $\alpha_{e q}$ is the equilibrium fractionation coefficient and $f_{e v}$ is the fraction of the rain that evaporates. Equation 2 tells us that the rain evaporation is more depleted as the rain is more depleted relative to the vapor (quantified by $\lambda$ ) and as the evaporated fraction $f_{e v}$ is smaller. This simple equation (Figure $7 \mathrm{~b}$, red) is able to approximate the simulated values of $\phi$ (black) for the ctrl simulation and is able to capture the smaller and larger values of $\phi$ for $\omega_{L S}-60$ and $\omega_{L S}+20$ respectively (Figure $7 \mathrm{c}-\mathrm{d}$ ).

We find that below the melting level, $\phi$ is smaller in $\omega_{L S}-60$ than in ctrl mainly because $f_{e v}$ is smaller (Figure $7 \mathrm{c}$, green). Near the melting level, $\phi$ is smaller in $\omega_{L S}-60$ than in ctrl both because $f_{e v}$ is smaller and because $\lambda$ is smaller, i.e. the rain is more depleted due to snow melt. In $\omega_{L S}+20$, the effect of $f_{e v}$ dominates at all levels.

\subsection{Summary}

To summarize, the previous sections suggest that rain evaporation in the lower troposphere is a key ingredient of the vapor amount effect. The isotopic composition of the rain evaporation flux mainly depends on the evaporated fraction of the rain (consistent with [Risi et al., 2008, Tremoy et al., 2014]). Near the melting level in regimes of large-scale ascent, it is also impacted by snow melt. We hypothesize that the isotopic effect of rain evaporation propagates downward down to the SCL. To test this hypothesis and to understand the underlying mechanisms, in the next section we develop a simple two-column model.

\section{A simple two-column model to quantify the relative contributions of different processes}

The previous section and previous studies provide a guide for developing our simple interpretative framework. First, the model needs to represent the effect of rain evaporation, highlighted as a key process in the previous section. Second, alternative hypotheses for the "vapor amount effect" have involve mixing between the subsident environment and detrained water ([Bailey et al., 2017], hypothesis 4). These processes also need to be represented in our model. Third, the steepness of the $q-\delta D_{v}$ relationship must be a key ingredient, since it drives $\delta D_{v}$ in the SCL and thus $\delta D_{v}$ everywhere. Finally, the previous section has relied on the distinction between the environment and cloudy regions. Keeping this distinction, we develop a two-column model.

\subsection{Model equations and numerical application to LES outputs}

\subsubsection{Balance equations}

This model is inspired by the two-column model used to predict tropospheric relative humidity in [Romps, 2014] and $\delta D$ profiles in [Duan et al., 2018]. The first column represents the cloudy regions, including cloudy updrafts and downdrafts, as a bulk entraining plume. The second column represents the subsident environment and precipitating downdrafts (Figure 8).

The mass balance for the air in the cloudy regions writes:

$$
\frac{d M}{d z}=M \cdot(\epsilon-\delta)
$$

where $M$ is the bulk mass flux in the cloudy regions (positive upward), $\epsilon$ and $\delta$ are the fractional entrainment and detrainment rates.

We assume that the specific humidity in the cloudy regions is at saturation, and call it $q_{s}$. The water balance in the cloudy regions writes:

$$
\frac{d\left(M q_{s}\right)}{d z}=\epsilon \cdot M \cdot q_{e}-\delta \cdot M \cdot q_{s}-c
$$

where $c$ is the condensation rate and $q_{e}$ is the specific humidity in the environment. The terms on the right hand side represent the water input by entrainment of environment air, the water loss by detrainment of cloudy air, and the water loss by condensation respectively. We assume that all the condensed water is immediately lost by the 
(a)

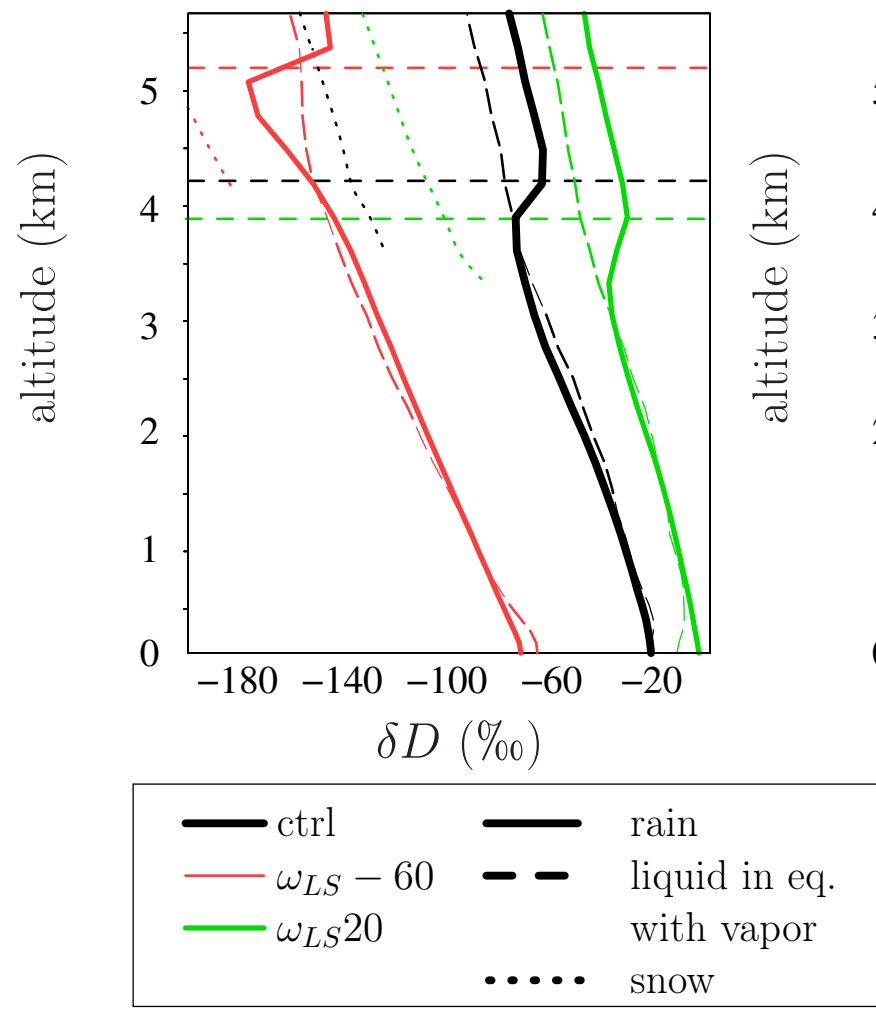

(c)

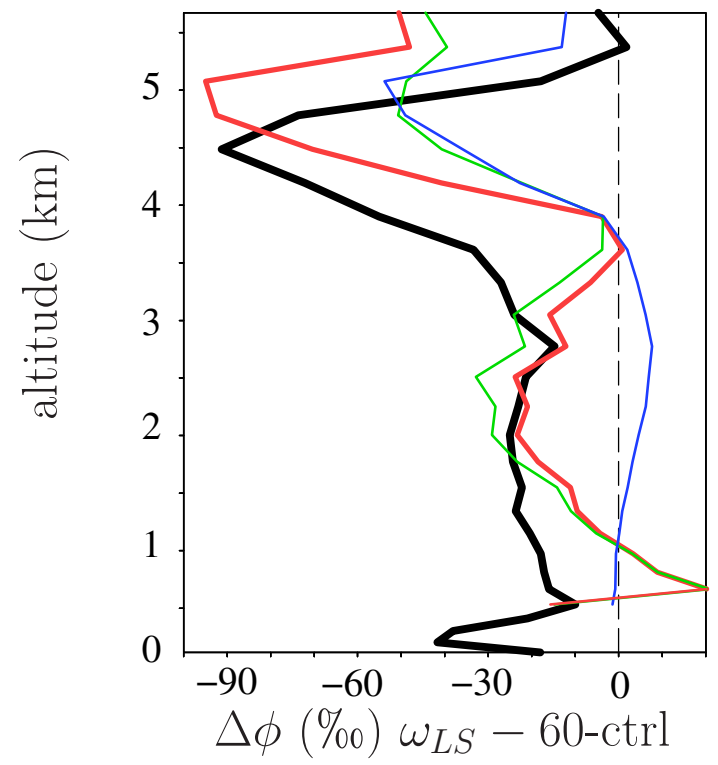

(b)

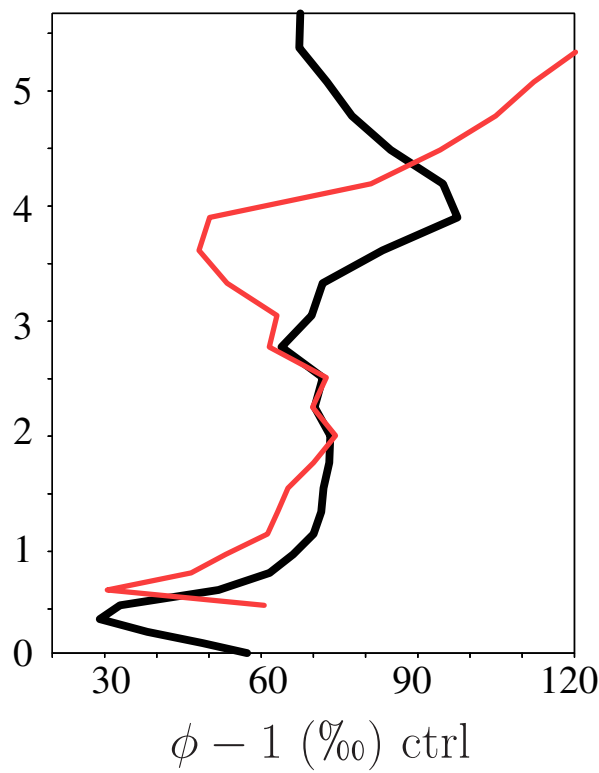

\begin{tabular}{|l|}
\hline - LES \\
$-\mathrm{Eq}$ \\
\hline
\end{tabular}

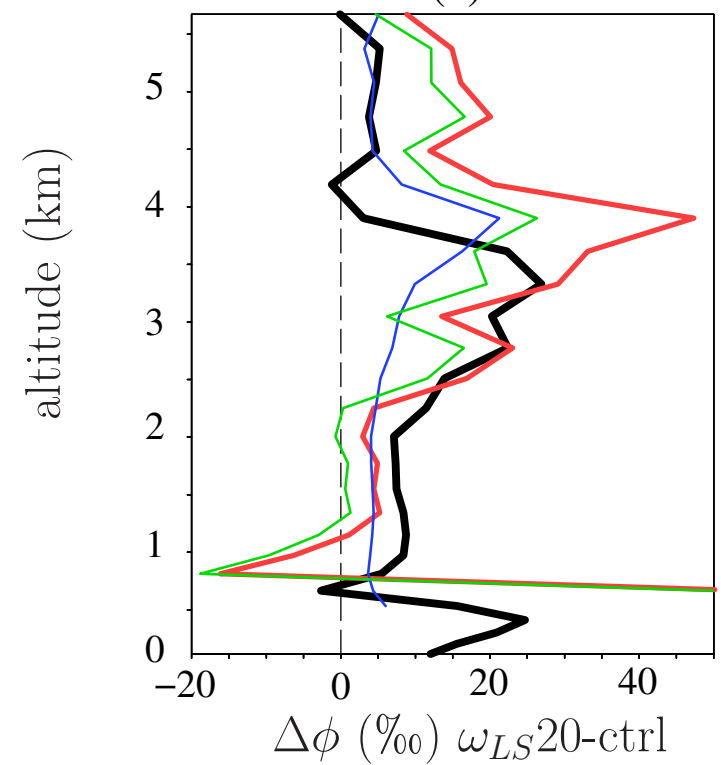

$$
\begin{aligned}
& \text { - LES } \\
& - \text { Eq } \\
& -f_{e v} \text { only } \\
& -\lambda \text { only }
\end{aligned}
$$

Figure 7: (a) $\delta D$ profile for rain water (solid) and snow (dotted) falling in the environment. The liquid that would be in equilibrium with the vapor in the environment is shown in dashed. (b) Profile of $\phi=R_{e v} / R_{e}$ simulated by the ctrl simulation (black, same as in Figure 6c black) and predicted by equation 2 (red). (c) Difference of $\phi$ between $\omega_{L S}-60$ and ctrl simulated by the LES (black), predicted by the equation 2 (red), predicted by equation 2 if only $f_{e v}$ varies (green) and if only $\lambda$ varies (blue). (d) Same as (c) but for the difference between $\omega_{L S} 20$ and ctrl. 


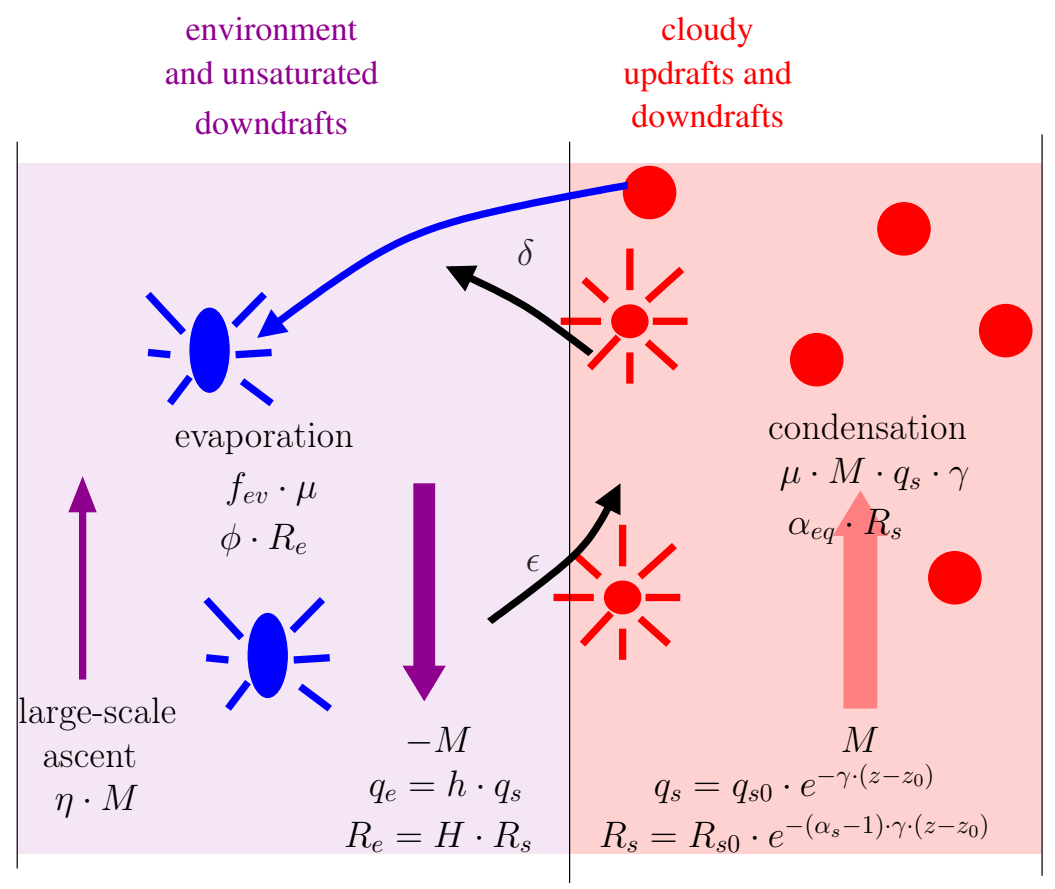

Figure 8: Schematic view of the simple two-column model, and definition of the main variables.

cloudy regions to the environment, and evaporation of this lost water can occur in the sub-saturated environment only (as in [Romps, 2014]).

We assume that mass is conserved within the domain, so that the flux in the environment is $-M$. The largescale ascent, when present, is taken into account through a humidity tendency, consistent with the LES set-up. We assume that the large-scale humidity tendency applies to the environment only, which is a first-order approximation justified by the small fraction of the domain that is covered by cloudy updrafts (less than 10\%). The water balance in the environment writes:

$$
\frac{d\left(-M q_{e}\right)}{d z}=-\epsilon \cdot M \cdot q_{e}+\delta \cdot M \cdot q_{s}+f_{e v} \cdot c-\eta \cdot M \cdot \frac{\partial q_{e}}{\partial z}
$$

where $f_{e v}$ is the fraction of the cloud or precipitating water that evaporates in the environment, $\eta=M_{L S} / M$ and $M_{L S}$ is the domain-mean large-scale mass flux. The terms on the right hand side represents the water loss by entrainment into cloudy regions, water input by the detrainment of cloudy air, partial evaporation of condensed water and water input by large-scale vertical advection.

Regarding water isotopes, we assume that the cloud water removed by condensation is in isotopic equilibrium with the cloudy region water vapor. The isotopic balance in the cloudy regions thus writes:

$$
\frac{d\left(M q_{s} \cdot R_{s}\right)}{d z}=\epsilon \cdot M \cdot q_{e} \cdot R_{e}-\delta \cdot M \cdot q_{s} \cdot R_{s}-c \cdot \alpha_{e q} \cdot R_{s}
$$

where $\alpha_{e q}$ is the equilibrium fractionation coefficient, $R_{s}$ is the isotopic ratio in the cloudy regions and $R_{e}$ is the isotopic ratio in the environment.

The isotopic balance in the environment writes:

$$
\frac{d\left(-M q_{e} \cdot R_{e}\right)}{d z}=-\epsilon \cdot M \cdot q_{e} \cdot R_{e}+\delta \cdot M \cdot q_{s} \cdot R_{s}+f_{e v} \cdot c \cdot \phi \cdot R_{e}-\eta \cdot M \cdot \frac{\partial\left(q_{e} R_{e}\right)}{\partial z}
$$

where recall that $\phi=R_{e v} / R_{e}$ and $R_{e v}$ is the ratio of the precipitation evaporation flux.

\subsubsection{Other simplifying assumptions and differential equations}

To simplify the equations, as in [Romps, 2014] we assume that $q_{s}$ is an exponential function of altitude:

$$
q_{s}=q_{s}\left(z_{0}\right) \cdot e^{-\gamma \cdot\left(z-z_{0}\right)}
$$


where $\gamma$ is a lapse rate in $m^{-1}$ calculated as $d \ln \left(q_{s}\right) / d z$.

For isotopes, we assume that the $R_{s}$ is a power function of $q_{s}$, consistent with a Rayleigh distillation:

$$
R_{s}=R_{s}\left(z_{0}\right)\left(q_{s} / q_{s 0}\right)^{a_{s}-1}
$$

Coefficient $\alpha_{s}$ represents the steepness of the $q-\delta D_{v}$ gradient in cloudy regions and remains to be estimated. As in [Duan et al., 2018], $R_{s}$ is thus an exponential function of altitude:

$$
R_{s}=R_{s}\left(z_{0}\right) \cdot e^{-\left(\alpha_{s}-1\right) \cdot \gamma \cdot\left(z-z_{0}\right)}
$$

We set:

$$
\begin{gathered}
q_{e}=h \cdot q_{s} \\
R_{e}=H \cdot R_{s}
\end{gathered}
$$

Combining equation 5 with equations 3 and 8 , we get the following differential equation for $h$ :

$$
\frac{\partial h}{\partial z}=h \cdot \gamma-\frac{\delta}{1-\eta}(1-h)-\frac{f_{e v} \cdot \mu \cdot \gamma}{1-\eta}
$$

where $\mu=c /\left(M \cdot q_{s} \cdot \gamma\right)$ represents the ratio of actual condensation $(c)$ relative to the condensation if the ascent was adiabatic $\left(M \cdot q_{s} \cdot \gamma\right)$. Similarly, combining equations 7 with equations 5 and 9 , we get the following differential equation for $H$ :

$$
\frac{\partial H}{\partial z}=H \cdot \gamma \cdot\left(\alpha_{s}-1\right)-\frac{\delta}{h \cdot(1-\eta)} \cdot(1-H)-\frac{f_{e v} \cdot \mu \cdot \gamma}{h \cdot(1-\eta)} \cdot H \cdot(\phi-1)
$$

We now have two equations with four unknowns: $h, H, \mu$ and $\alpha_{s}$. The condensation efficiency $\mu$ can be deduced from equations 4 :

$$
\mu=1-\frac{\epsilon}{\gamma} \cdot(1-h)
$$

This equation, similar to one in [Romps, 2014], reflects the fact that condensation efficiency decreases when entrainment $\epsilon$ increases and when the entrained air is drier. If $\epsilon=0$ or $h=1$, then $\mu=1$.

Similarly, the $q-\delta D_{v}$ steepness $\alpha_{s}$ in cloudy air can be deduced from equation 6 :

$$
\alpha_{s}-1=\mu \cdot\left(\alpha_{e q}-1\right)+\frac{\epsilon}{\gamma} \cdot h \cdot(1-H)
$$

This equation tells us that two effects control the steepness of the $q-\delta D_{v}$ gradient. First, there is a "dilution effect": if dry air is entrained, then the condensation efficiency $\mu$ decreases. This reduces $\alpha_{s}$ compared to $\alpha_{e q}$, i.e. compared to what we would expect from Rayleigh distillation. Second, there is an "isotopic contrast effect": if depleted water vapor is entrained $(H<1)$ is entrained, then $\alpha_{s}$ becomes steeper. This is how a depleting effect of rain evaporation can translate into a larger steepness, and eventually more depleted SCL.

\subsubsection{Numerical solutions}

To get analytical solutions for $h$ and $H$, [Romps, 2014] and [Duan et al., 2018] assume that $h \cdot \frac{\partial q_{s}}{\partial z} \gg q_{s} \cdot \frac{\partial h}{\partial z}$ and that $H \cdot \frac{\partial R_{s}}{\partial z} \gg R_{s} \cdot \frac{\partial H}{\partial z}$. This allows them to calculate $h$ and $H$ as the solution of a simple linear equation and of a second order polynomial respectively. However, there are two issues with these solutions. First, although these solutions behave reasonably for $h$ ( [Romps, 2014]), they become very noisy, unstable or unrealistic for values of $\epsilon, \delta$ and $f_{e v}$ that are diagnosed from LES outputs. This is because there is a powerful positive feedback between $\alpha_{s}$ and $H$ : as $H$ decreases, more depleted vapor is entrained in updrafts which increases the steepness $\alpha_{s}$; in turn, the stronger steepness $\alpha_{s}$ makes the subsidence more efficient at depleting the environment, further decreasing $H$. [Duan et al., 2018] circumvented this problem by assuming $\epsilon$ and $\delta$ that are constant with altitude and equal to each other, but it is at the cost of artificially reducing freedom for the solutions. Second, our hypothesis is that rain evaporation near the melting level affects the isotopic profiles down to the SCL. We thus want each altitude to feel the memory of processes at higher altitudes. The term with $\frac{\partial H}{\partial z}$ is thus a key ingredient in our framework.

Therefore, we choose to numerically solve the differential equations 10 and 11 . We start from an altitude of $5 \mathrm{~km}$ with $h=0.8$ and $H-1=-10 \%$. We do not start above $5 \mathrm{~km}$ because entrainment is more difficult to 
diagnosed above the melting level (section 3.1.4). We integrate equations 10 and 11 down to the SCL top around $500 \mathrm{~m}$. The resulting $h$ profile is a function of the profiles of 5 input parameters: $\gamma, \epsilon, \delta, f_{e v}$ and $\eta$. The $H$ profile is a function of 7 input parameters: $\gamma, \epsilon, \delta, f_{e v}, \eta, \alpha_{e q}$ and $\phi$. These input parameters are all diagnosed from the LES simulations as detailed below. In each LES level, the input parameters are assumed constant and equations 10 and 11 are integrated within each layer over 50 sub-layers.

\subsubsection{Diagnosed input parameters}

Parameters $f_{e v}, \alpha_{e q}$ and $\phi$ were already plotted in Figure 6 and discussed in section 2.6. Parameter $\gamma$ is calculated from domain-mean profiles. It is steeper in ctrl than in $\omega-60$ because of the steeper temperature gradient resulting from the drier air (Figure 9a). Parameter $\eta=M_{L S} / M$ is calculated from the net upward mass flux in cloudy regions $M$ (Figure 9b), which is calculated as the average vertical velocity in cloudy regions multiplied by the area fraction of the cloudy region. Entrainment $\epsilon$ is diagnosed by using the conservation of the frozen moist static energy (e.g. [Hohenegger and Bretherton, 2011, Del Genio and Wu, 2010]):

$$
\frac{\partial m_{s}}{d z}=\epsilon \cdot\left(m_{e}-m_{s}\right)
$$

where $m_{s}$ and $m_{e}$ are the frozen moist static energy in the convective region and the environment respectively. The application of this equation is limited to the lower troposphere. Above the melting level, we would need to account for the precipitation of ice ([Pauluis and Mrowiec, 2013]) and for the lofting of rain. Therefore, we arbitrarily set $\epsilon=0.5 \mathrm{~km}^{-1}$ above the melting level. Entrainment is maximal in the sub-cloud layer, and decreases exponentially with height (Figure 9c), consistent with previous studies ([Del Genio and Wu, 2010, De Rooy et al., 2013]).

Finally, detrainment $\delta$ is deduced from $\epsilon$ and $M$ using equation 3. Detrainment shows the typical trimodal distribution ([Johnson et al., 1999]) (Figure 9d), with a first maximum just above the SCL top corresponding to the detrainment of shallow convection, a second maximum near the melting level near $4.5 \mathrm{~km}$ corresponding to the detrainment of congestus convection, and a third maximum in the upper troposphere corresponding to the deep convection (not shown in 9d).

\subsubsection{Closure in the sub-cloud layer}

To calculate the full $\delta D$ profiles, we need as initial condition the isotopic ratio in the SCL. With this aim, we use a simplified version if the sub-cloud layer model of [Risi et al., 2020]. We assume that water enters the SCL through surface evaporation and through downdrafts at the SCL top, and exits the SCL through updrafts at the SCL top. We neglect large-scale forcing and rain evaporation, since they have a small impact in the SCL ([Risi et al., 2020]). The air flux of updrafts equals that of downdrafts. We define $r_{u}=q_{u} / q_{1}$ and $r_{d}=q_{d} / q_{1}$, where $q_{1}$ is the mixing ratio in the SCL and $q_{u}$ and $q_{d}$ are the mixing ratios in updrafts and downdrafts at the SCL top. We assume that the water vapor is more enriched as the air is moister, following a logarithmic function: $R_{u}=R_{1} \cdot r_{u}^{\alpha_{u}-1}$ and $R_{d}=R_{1} \cdot r_{d}^{\alpha_{d}-1}$ where $R_{u}$ and $R_{d}$ are isotopic ratios in updrafts and downdrafts, and $\alpha_{u}$ and $\alpha_{d}$ are $q-\delta D$ steepness for updrafts and downdrafts. Water and isotopic budgets yield:

$$
R_{1}=\frac{R_{o c e} / \alpha_{e q}(S S T)}{h_{1}+\alpha_{K} \cdot\left(1-h_{1}\right) \cdot \frac{r_{u}^{\alpha_{u}}-r_{d}^{\alpha_{d}}}{r_{u}-r_{d}}}
$$

where $R_{\text {oce }}$ is the isotopic ratio at the ocean surface, $\alpha_{e q}(S S T)$ is the equilibrium fractionation coefficient at the sea surface temperature, $\alpha_{K}$ is kinetic fractionation coefficient ([Merlivat and Jouzel, 1979]) and $h_{1}$ is the relative humidity normalized at the SST and accounting for ocean salinity: $h_{1}=q_{1} / q_{\text {sat }}^{\text {surf }}(S S T), q_{\text {sat }}^{\text {surf }}(S S T)=$ $0.98 \cdot q_{s a t}(S S T)$ and $q_{s a t}$ is the humidity saturation as a function of temperature at the sea level pressure. We assume $\delta D_{\text {oce }}=0 \%$ and $h_{1}$ is diagnosed from the LES.

For $r_{u}$ and $r_{d}$, we use values for the ctrl simulation, because small changes in $r_{u}$ and $r_{d}$ across simulations have only a marginal impact on $R_{1}$ ([Risi et al., 2020]). Following [Risi et al., 2020], we set $r_{u}-1=1.44 \%$ and $r_{d}-1=-0.38 \%$. For $\alpha_{u}$ and $\alpha_{d}$, [Risi et al., 2020] had shown that they scale with $\alpha_{z}$ values just above the SCL top, but with larger values especially for simulations with large-scale ascent. We use an empirically-fitting function: $\alpha_{u}=\alpha_{d}=1+100 \cdot\left(\widetilde{\alpha_{z}}-1\right)^{3}$, where $\widetilde{\alpha_{z}}=1+\frac{\ln \left(R\left(z_{S C T}\right) / R\left(z_{S C T}+1 \mathrm{~km}\right)\right)}{\ln \left(q\left(z_{S C T}\right) / d\left(z_{S C T}+1 \mathrm{~km}\right)\right)}$ where $z_{S C T}$ is the SCL top.

Finally, since the updraft region covers only a very small fraction of the domain, we assume that $R_{e}\left(z_{S C T}\right) \simeq R_{1}$. The procedure to calculate the full $\delta D_{v}$ profiles is as follows:

1. vertical profiles for $h, H$ and $\alpha_{s}$ are calculated through a downward integration of equations 10-13 following 3.1.3. 
(a)

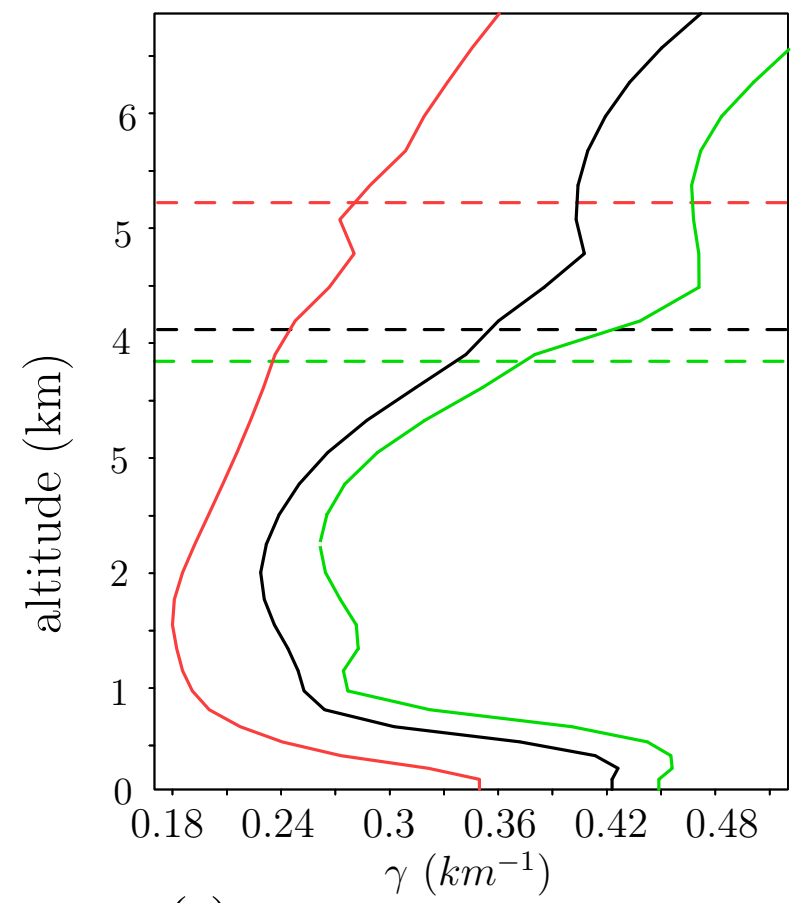

(c)

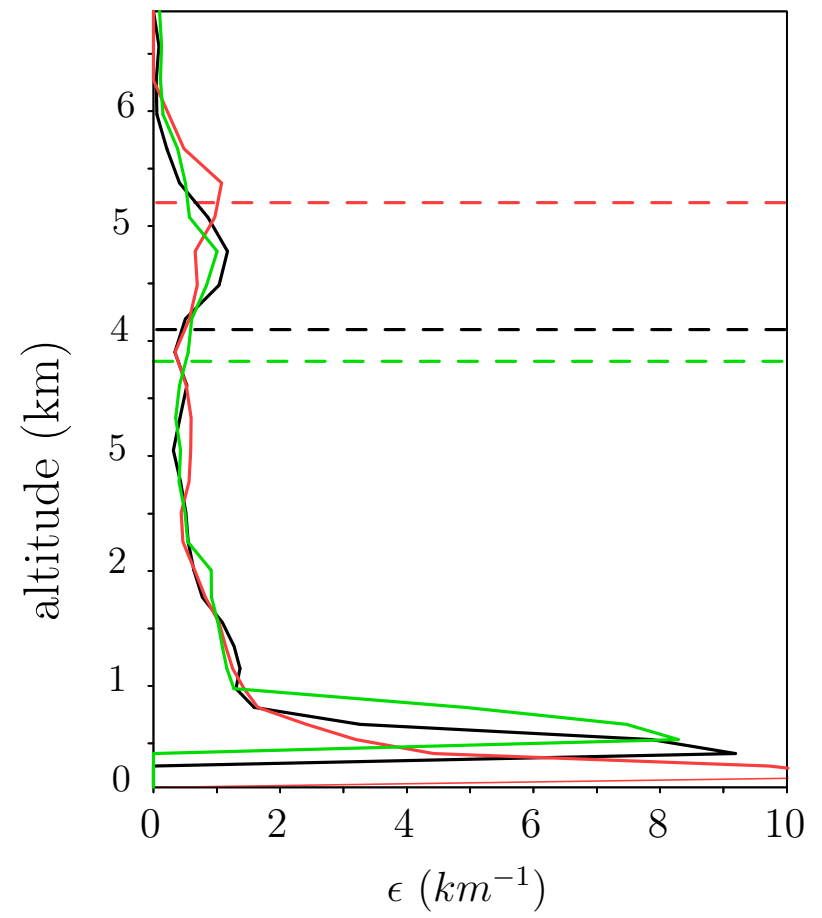

(b)

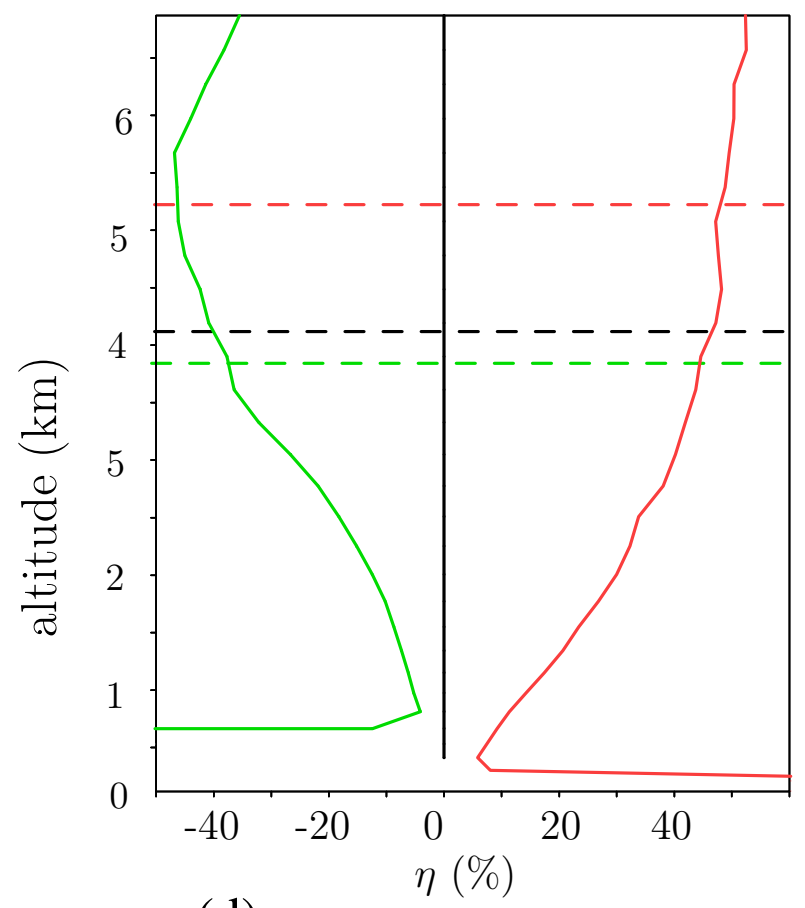

(d)

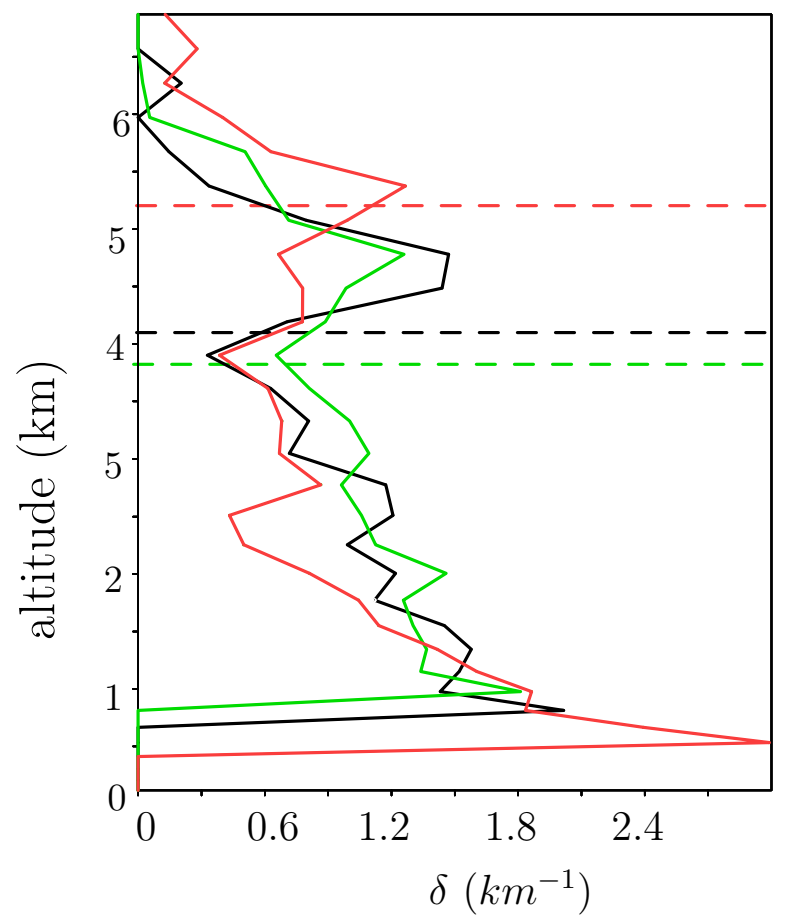

$$
\begin{aligned}
& -\operatorname{ctrl} \\
& -\omega_{L S}-60 \\
& -\omega_{L S} 20
\end{aligned}
$$

Figure 9: Input parameters for the simple model, for ctrl (black), $\omega_{L S}-60$ (red) and $\omega_{L S}+20$ (green). (a) saturation specific humidity lapse rate $\gamma$; (b) ratio of large-scale vertical mass flux over the convective cloud mass flux; (c) entrainment rate; (d) detrainment rate. 
2. The vertical profile for a normalized version of $R_{s}, R_{s, \text { norm }}$ that satisfies $R_{s, \text { norm }}\left(z_{S C T}\right)=1$, is calculated based on the $\alpha_{s}$ profile through an upward integration.

3. The vertical profile for a normalized version of $R_{e}, R_{e, n o r m}$, is calculated as $R_{e, \text { norm }}=R_{s, \text { norm }} \cdot H$.

4. From the $R_{e, n o r m}$ profile, $\widetilde{\alpha_{z}}$ is estimated.

5. From $h_{1}$ and $\widetilde{\alpha_{z}}, R_{1}$ is estimated.

6. The full $R_{e}$ profile can finally be calculated so that $R_{e}\left(z_{S C T}\right) \simeq R_{1}: R_{e}=R_{e, n o r m} \cdot R_{1} / H\left(z_{S C T}\right)$.

\subsubsection{Evaluation of the two-column model}

The two-column model successfully captures the order of magnitude and the shape of the vertical profile of relative humidity for the ctrl simulation (Figure 10a), as well as the moister troposphere in $\omega_{L S}-60$ and the drier troposphere in $\omega_{L S}+20$ (Figure 10b-c).

It successfully captures the vertical profile of $\delta D_{v}$ (Figure 10b) and the more depleted troposphere in $\omega_{L S}-60$ but underestimate the $\delta D_{v}$ difference by about half (Figure 10e). It also captures the more enriched troposphere in $\omega_{L S}+20$ but again underestimate the $\delta D_{v}$ difference especially in the middle troposphere (Figure 10f). Similarly, it approximately captures the steepness $\alpha_{z}$ and the sign of the $\alpha_{z}$ differences across simulations, but underestimates the $\alpha_{z}$ differences (Figure 10g-i).

These mismatches are caused by mismatches in the estimate of the relative enrichment of the environment relative to the updrafts $H$. Although it is reasonably well predicted for the ctrl simulation (Figure 10c), the model fails to simulate the smaller $H$ for $\omega_{L S}-60$ in the middle troposphere and the larger $H$ for $\omega_{L S}+20$ almost everywhere. The two-column model overestimates the impact of $\eta$ and predicts a behavior for $H$ that is too similar to that of $h$. We could not find the exact reason for this shortcoming, but we have to acknowledge that the two-column model hides many horizontal heterogeneities. We will have to keep this shortcoming in mind when interpreting the results.

\subsection{Decomposition of relative humidity and $\delta D$ variations}

To estimate the impact of the different input parameters on the relative humidity profile $h$, we modify them one by one from the ctrl simulation to the $\omega_{L S}-60$ and from the ctrl simulation to $\omega_{L S}+20$ simulations.

\subsubsection{Decomposition of relative humidity}

The moister troposphere in $\omega_{L S}-60$ is mainly due to the larger $\eta$, i.e. the direct moistening effect of large-scale ascent (Figure 11a). The thermodynamic structure, entrainment, detrainment and rain evaporation have a much smaller effect. Similarly, The drier troposphere in $\omega_{L S}+20$ is mainly due to the more negative $\eta$, i.e. the direct drying effect of large-scale descent (Figure 11b).

Note that the direct effect of $\eta$ on environment relative humidity may be an artifact of the way the largescale forcing is prescribed in our LES. The large-scale circulation is accounted for through tendencies in humidity, temperature and isotopes that are horizontally homogeneous. Therefore, since the environment covers most of the domain, most of the large-scale ascent or descent is felt in the environment. In reality, in case of large-scale ascent for example, we expect the environment to keep the same subsidence rate, corresponding to radiative subsidence. Rather, we expect the large-scale ascent to be absorbed by the larger cloud fraction ([Emanuel et al., 1994]). In our interpretative framework, this would be equivalent to adding the large-scale in the cloudy column rather than in the environment. This would increase the condensation efficiency $\mu$, and would eventually moisten the environment through rain evaporation, but less than if the large-scale ascent was homogeneous (Text S3). We have to keep this in mind if trying to connect the sensitivity of $h$ to large-scale circulation in our LES and in reality.

\subsubsection{Dilution effect on $\delta D$}

A first effect impacting $\delta D_{v}$ profiles is the dilution by entrainment (section 3.1.2). In the absence of entrainment $(\epsilon=0)$, the steepness in the updraft column would be $\alpha_{s}=\alpha_{e q}$ (Figure 12a, black). Because dry air is entrained, the condensation rate is reduced by the factor $\mu$ following equation 12. According to equation 13, this reduces the steepness (Figure 12a, green). This effect of entrainment can be understood as a mixing process: as the air rises and condensation proceeds, the remaining air is mixed with dry air from entrainment and with droplets that evaporate. Consistent with the convex shape of the mixing lines, this leads to a reduction of the $q-\delta D$ steepness (Figure 1). 

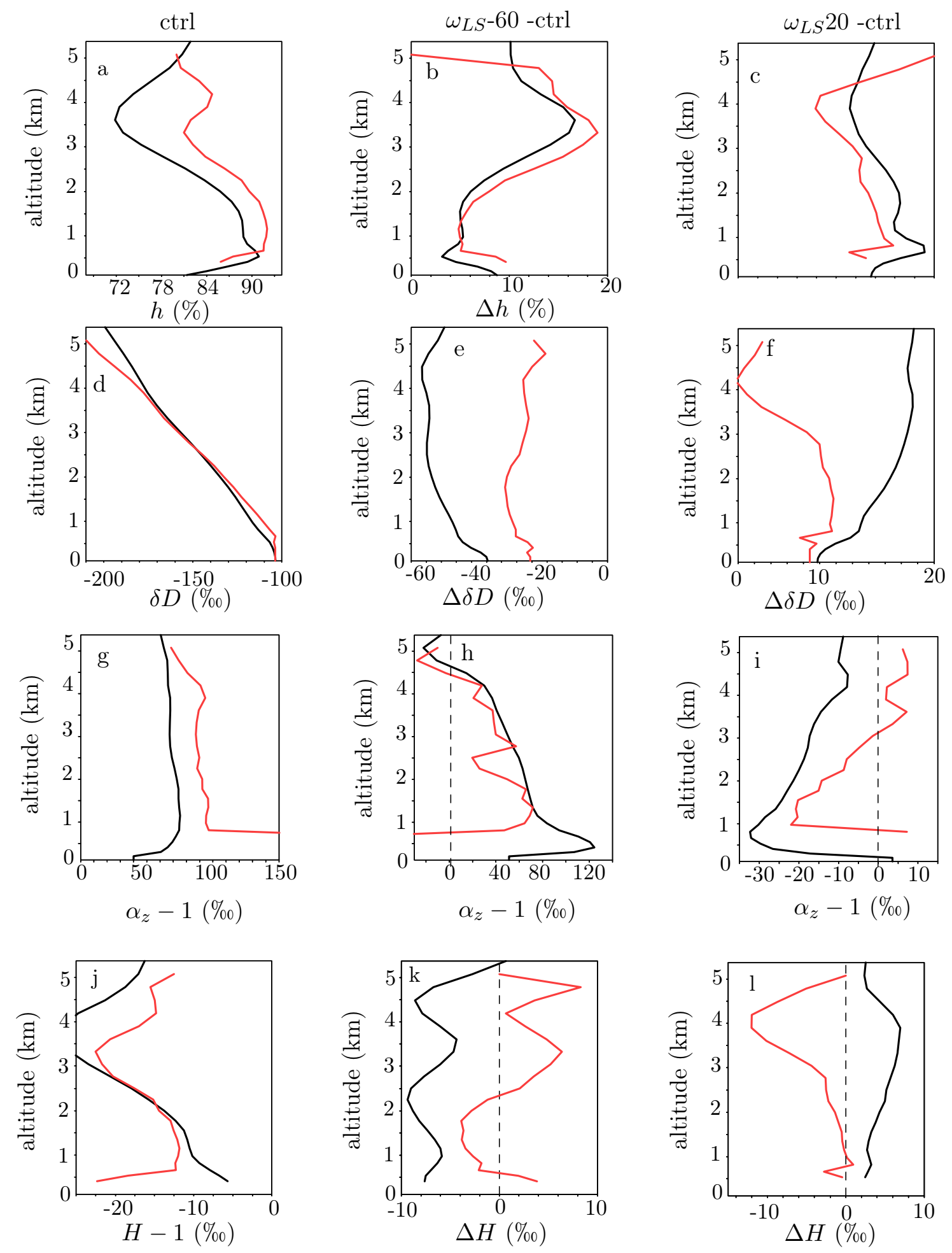

\section{— simulated by the LES \\ — predicted by the two-column model}

Figure 10: (a) Relative humidity $h$ simulated by the LES (black) and predicted by the two-column model (red) for the ctrl simulation. (b) Same as (a) but for the difference between $\omega_{L S}-60$ and ctrl. (c) Same as (b) but for the difference between $\omega_{L S}+20$ and ctrl. (d-f) Same as (a-c) but for the water vapor $\delta D$. (g-i) Same as (a-c) but for the steepness $\alpha_{z}$. (j-1) Same as (a-c) but for the relative enrichment of the environment relative to the updrafts $H$. 
(a) $\omega_{L S}-60-\operatorname{ctrl}$

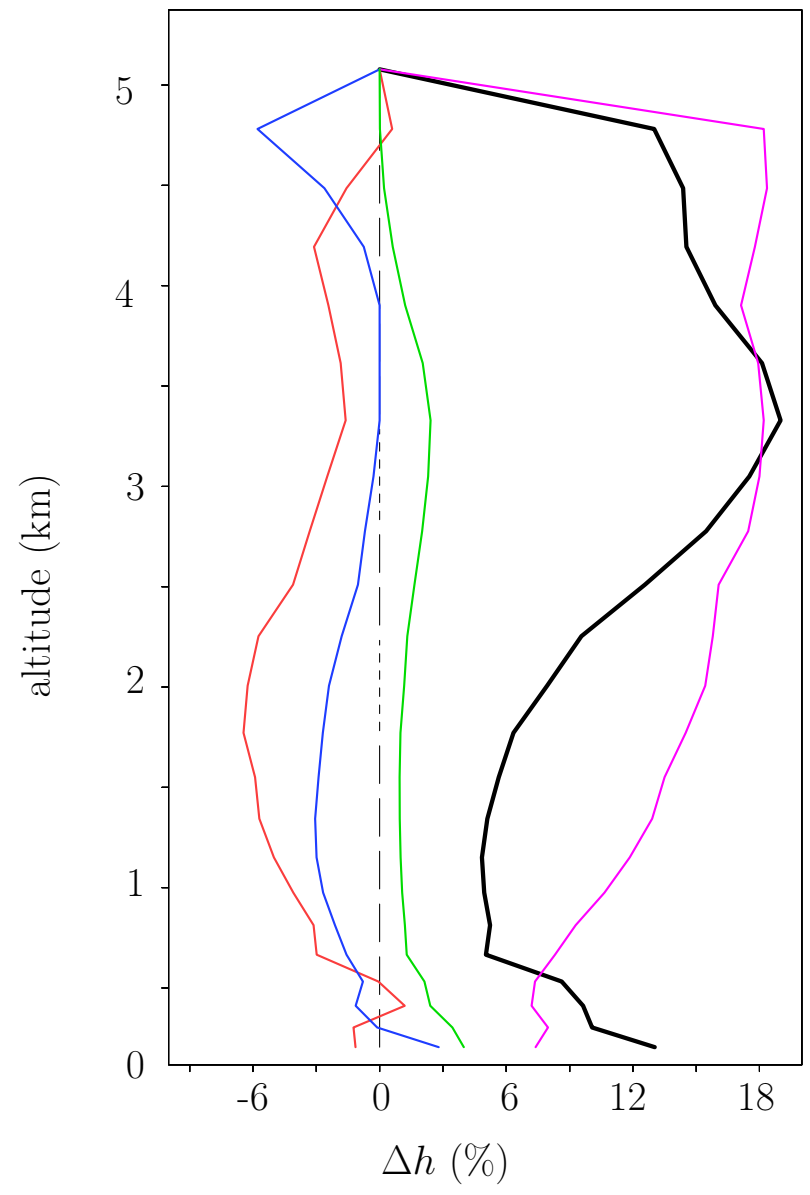

(b) $\omega_{L S}+20-$ ctrl

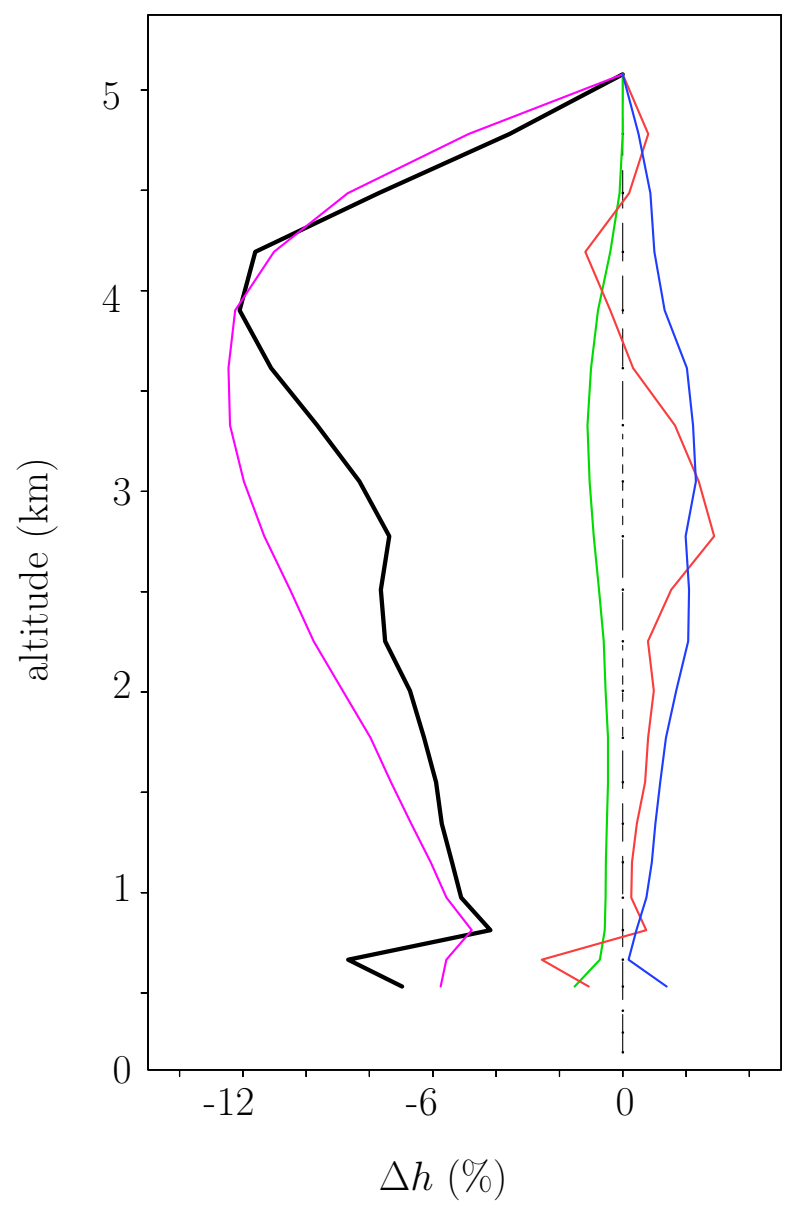

$$
\begin{array}{ll}
- & \text { total } \\
& \text { effect of } \eta \text { (large-scale forcing) } \\
& \text { effect of } \gamma \text { (thermodynamic structure) } \\
& \text { effect of } \epsilon \text { and } \delta \text { (entrainment and detrainement) } \\
& \text { effect of } f_{e v} \text { (rain evaporation) }
\end{array}
$$

Figure 11: (a) Relative humidity difference between $\omega_{L S}-60$ and ctrl predicted by the two-column model (black) and its contributions from variations of input parameters one by one: $\eta$ (pink), $\gamma$ (green), $\epsilon$ and $\delta$ (red) and $f_{e v}$ (blue). (b) Same as (a) but for the difference between $\omega_{L S}+20$ and ctrl. 
(a)

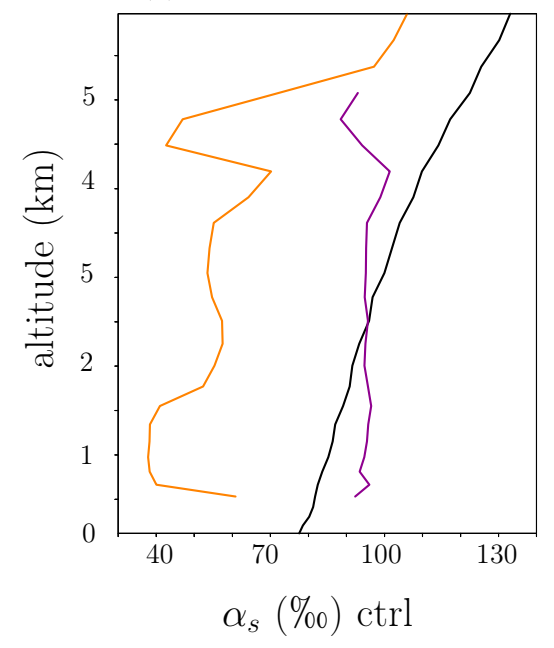

- if $h=1$ and $H=1$

- if $H=1$

- full equation (b)

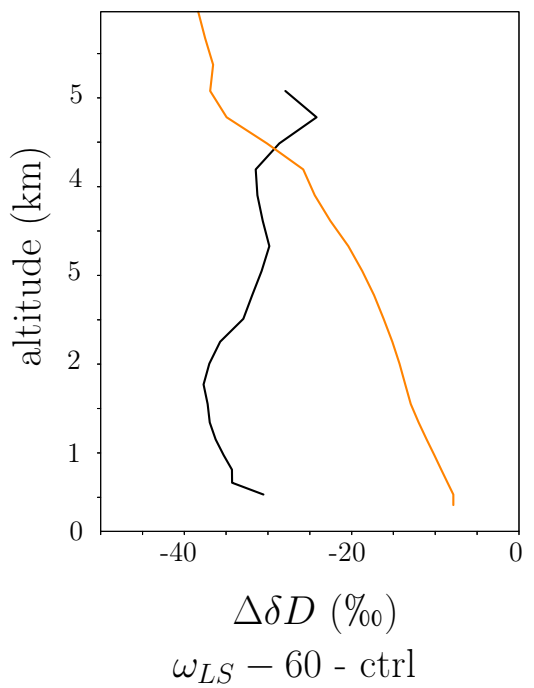

(c)

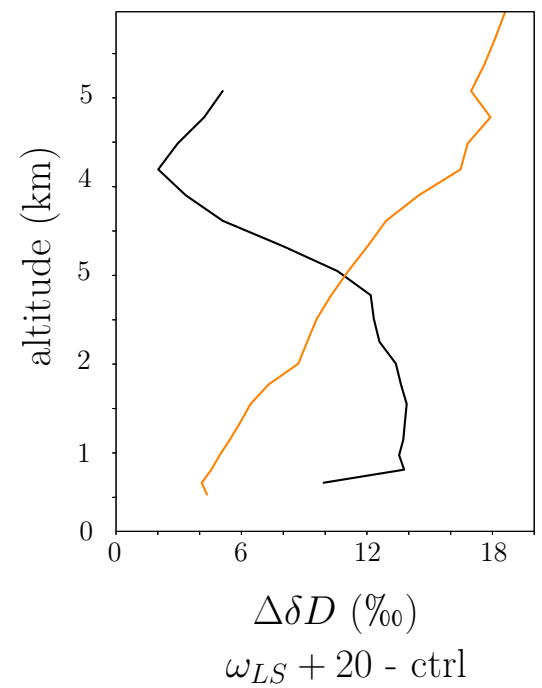

Figure 12: (a) Fractionation coefficient $\alpha_{e q}$ (black), corresponding to the steepness in the updraft column $\alpha_{s}$ if $h=1$ and $H=0$; steepness $\alpha_{s}$ predicted if $h<1$ and $H=0\left(\alpha_{s}=1+\mu \cdot\left(\alpha_{e q}-1\right)\right)$ (green); steepness $\alpha_{s}$ from the full equation 13 (red). (b) Difference in $\delta D_{v}$ from ctrl to $\omega_{L S}-60$ predicted by the two-column model (red) and predicted if accounting only for the dilution effect (green). (c) Same as (b) but for $\omega_{L S}+20$.

\begin{tabular}{|c|c|c|}
\hline Difference from ctrl & $\omega_{L S}-60$ & $\omega_{L S}+20$ \\
\hline \hline Total simulated by the LES (\%) & -40 & 10 \\
\hline Total predicted by the two-column model (\%) & -30 & 11 \\
\hline Dilution effect (\%o, \%) & $-9(29 \%)$ & $5(47 \%)$ \\
\hline
\end{tabular}

Table 1: Difference of $\delta D_{v}$ between $\omega_{L S}-60$ and ctrl and between $\omega_{L S}+20$ and ctrl simulated by the LES and predicted by the two-column model, and the contribution of the dilution effect.

As a consequence of this "dilution effect", tropospheric $\delta D_{v}$ is less depleted than predicted by Rayleigh distillation. Since the troposphere is moister in $\omega_{L S}-60$, this "dilution effect" contributes to the more depleted $\delta D_{v}$ in $\omega_{L S}-60$ (Figure 12b, green). Reciprocally, since the troposphere is drier in $\omega_{L S}+20$, this "dilution effect" contributes to the more enriched $\delta D_{v}$ in $\omega_{L S}+20$ (Figure 12c, green). Quantitatively, the contribution of this dilution effect on the SCL $\delta D_{v}$ difference is $29 \%$ for $\omega_{L S}-60$ and $47 \%$ for $\omega_{L S}+20$ (table 2). The contribution increases with altitude.

Note that the two-column model likely overestimates this contribution, because of the shortcoming mentioned in section 3.1.6. The fact that only one third of the $\delta D_{v}$ difference remains when post-condensation effects are turned off confirms that these contributions are overestimated.

\subsubsection{Decomposition of $\delta D$}

In $\omega_{L S}-60$, the more depleted troposphere is driven primarily by the effect of the smaller $\phi$, i.e. the more depleted rain evaporation (Figure 13a, cyan). It explains $147 \%$ of the $\delta D_{v}$ difference in the SCL (Table 2). The smaller rain evaporated fraction (smaller $f_{e v}$ ) is the second main contributor (Figure 13a, blue, $43 \%$ in the SCL). This positive contribution is explained by the fact that evaporation has an overall enriching effect. The third main contributor is the larger $\eta$ (i.e. large-scale ascent), contributing to $26 \%$ of the $\delta D_{v}$ difference. This contribution corresponds mainly to the "dilution effect" explained in section 3.2.2. The sum of these contributions exceeds 100\%, because there are some dampening effects, especially $h_{1}$ : the moister surface relative humidity reduces the kinetic fractionation during surface evaporation.

In $\omega_{L S}+20, \eta$ becomes the main contribution to the $\delta D_{v}$ difference in the SCL (126\%), associated with the 
(a) $\omega_{L S}-60-\operatorname{ctrl}$

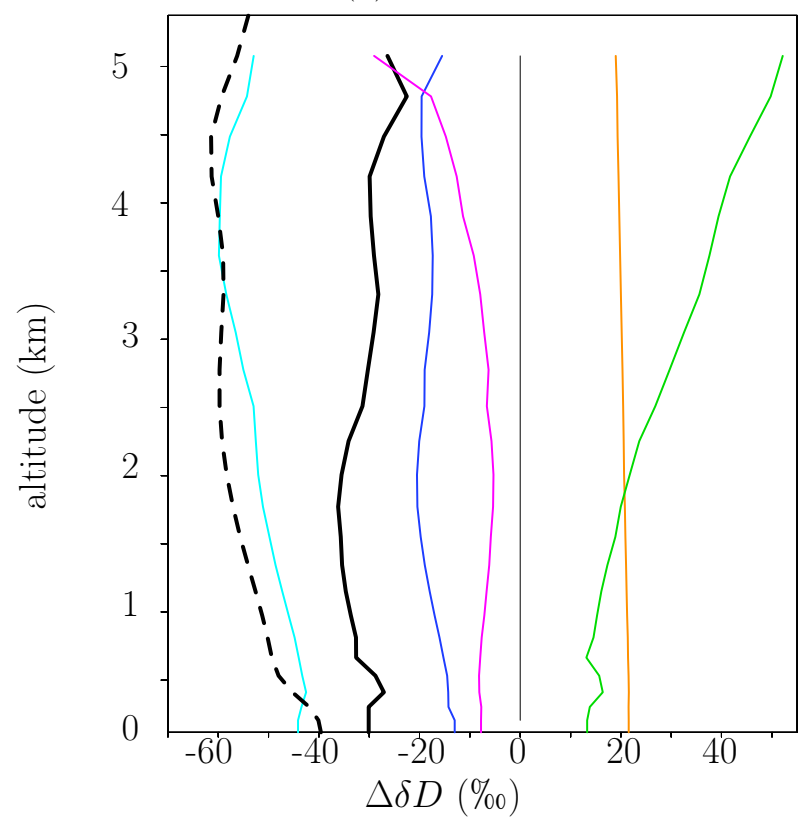

(b) $\omega_{L S}+20-$ ctrl

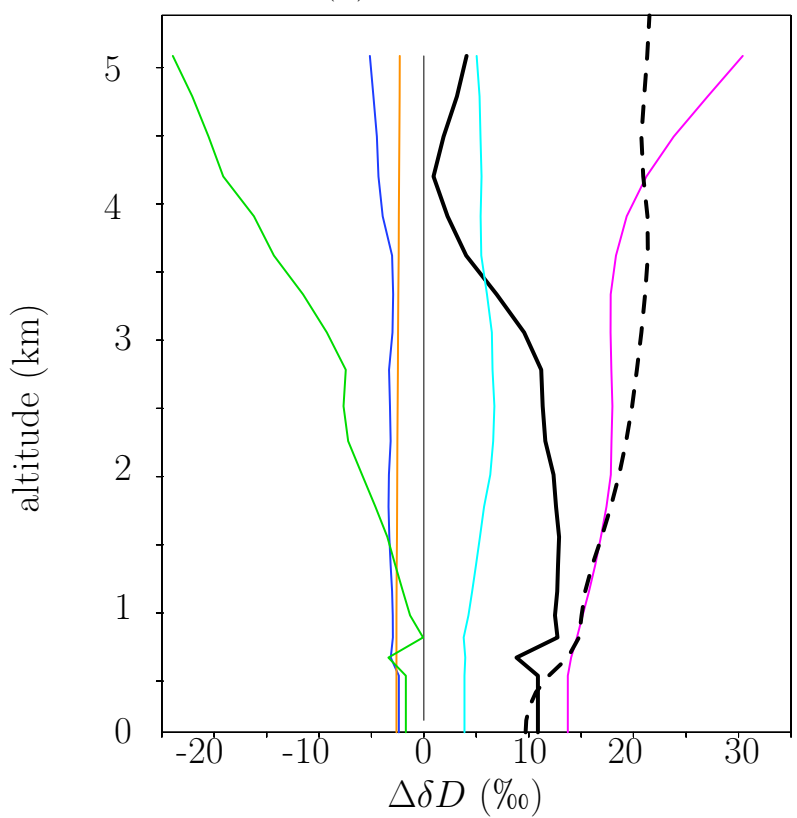

- - simulated by LES

— predicted by simple model

— effect of $\eta$ (large-scale forcing)

effect of $\gamma, \alpha_{e q}, \epsilon, \delta$ (thermodynamic structure, entrainment,detrainment)

effect of $f_{e v}$ (rain evaporation)

effect of $\phi$ (rain evaporation composition)

effect of $\phi$ (rain evaporation composition)

Figure 13: (a) $\delta D_{v}$ difference between $\omega_{L S}-60$ and ctrl predicted by the two-column model (black) and its contributions from variations of input parameters one by one: $\eta$ (pink), $\gamma$ and $\alpha_{e q}$ (green), $\epsilon$ and $\delta$ (red), $f_{e v}$ (blue), $\phi$ (cyan) and $h_{1}$ (orange). (b) Same as (a) but for the difference between $\omega_{L S}+20$ and ctrl.

dilution effect. The effect of the larger $\phi$, i.e. the more enriched rain evaporation, contributes to $36 \%$ to the $\delta D_{v}$ difference in the SCL.

This decomposition can be reconciled with the result that about one third of the $\delta D_{v}$ difference from ctrl to $\omega_{L S}-60$ remains when the fractionation during condensate evaporation is de-activated. This remaining difference is associated with (1) the dilution effect, and (2) the portion of the $\phi$ contribution that is due to the more depleted rain due to more snow melt. The fact that the sum of this two contributions exceeds one third suggests that the underestimate of $\delta D_{v}$ variations by the simple model is due to the underestimate of the effect of rain evaporation.

We note that the relative contributions of the different processes are very homogeneous in the vertical. For example, in the SCL, half of the contribution of $\phi$ comes from $\phi$ above $3 \mathrm{~km}$. This shows the strong "memory" of water vapor $\delta D$, which integrates processes downwards in the environment column, and then upward in the updraft column.

\section{Conclusion}

\subsection{Summary}

This study gives a comprehensive and quantitative understanding of the processes underlying the vapor amount effect, at least in our LES simulations. This understanding is illustrated in Figure 14:

1. When the troposphere is moister (in terms of relative humidity), less snow sublimates and thus more snow is available for melting. Snow melt results in rain that is more depleted relative to a liquid in equilibrium with 


\begin{tabular}{|c|c|c|}
\hline SCL $\delta D_{v}$ difference from ctrl & $\omega_{L S}-60$ & $\omega_{L S}+20$ \\
\hline \hline Total simulated by the LES (\%) & -40 & 10 \\
\hline Total predicted by the two-column model $(\%)$ & -30 & 11 \\
\hline Effect of $\gamma$ and $\alpha_{e q}(\%, \%)$ & $8(-25 \%)$ & $-3(-30 \%)$ \\
\hline Effect of $\epsilon$ and $\delta(\%, \%)$ & $6(-19 \%)$ & $2(14 \%)$ \\
\hline Effect of $\eta(\%, \%)$ & $-8(26 \%)$ & $14(126 \%)$ \\
\hline Effect of $f_{e v}(\%, \%)$ & $-13(43 \%)$ & $-2(-22 \%)$ \\
\hline Effect of $\phi(\%, \%)$ & $-44(147 \%)$ & $4(36 \%)$ \\
\hline Including $\phi$ above $3 \mathrm{~km}(\%, \%)$ & $-23(76 \%)$ & $2(23 \%)$ \\
\hline Effect of $h_{1}(\%, \%)$ & $22(-72 \%)$ & $-3(-24 \%)$ \\
\hline
\end{tabular}

Table 2: Difference of $\delta D_{v}$ in the SCL between $\omega_{L S}-60$ and ctrl and between $\omega_{L S}+20$ and ctrl simulated by the LES and predicted by the two-column model, and the contribution of different effects. The sum of all the different effects, except the line "Including $\phi$ above $3 \mathrm{~km}$ ", is $100 \%$ of the predicted $\delta D_{v}$ difference. The line "Including $\phi$ above $3 \mathrm{~km}$ " is a part of "Effect of $\phi$ "

the vapor, which leads to more depleted rain evaporation flux. When the troposphere is moister, the rain evaporated fraction is also smaller, making the rain evaporation flux even more depleted.

2. The more depleted evaporation depletes the environment more efficiently relative to updrafts. When this more depleted environment is entrained into the updrafts, it makes the vertical $q-\delta D$ gradient steeper. In turn, the steeper $q-\delta D$ vertical gradient makes the subsidence more efficient at depleting the environment, in a positive feedback that makes the vertical $q-\delta D$ gradient even steeper. Overall, this mechanisms allows to propagate the isotopic anomalies associated with rain evaporation downwards.

3. When the troposphere is moister, the updrafts get less diluted as they rise. They condense water vapor more efficiently, which also contributes to the steeper vertical $q-\delta D$ gradient.

4. The steeper gradient in the lower troposphere makes updrafts and downdrafts at the SCL top more efficient in depleting the SCL water vapor ([Risi et al., 2020]).

5. Finally, since the SCL serves as the initial condition for the full $\delta D$ vertical profiles, the water vapor is more depleted at all altitudes in the troposphere.

Coming back to our initial hypotheses, the dominant role of rain evaporation and rain-vapor diffusive exchanges confirm hypothesis 3 ([Lawrence et al., 2004, Risi et al., 2008, Lee and Fung, 2008]). The role of entrainment in diluting updrafts and reducing their condensation efficiency is reminiscent of hypothesis 4.

We notice that the root of the amount effect in the water vapor is higher relative humidity, with a triple effect on reducing sublimation, reducing the rain evaporated fraction and reducing the dilution of updrafts by entrainment. This explains why the amount effect can be observed only when the precipitation increase is associated with a change in the large-scale circulation ([Bony et al., 2008, Moore et al., 2014, Bailey et al., 2017, Risi et al., 2020]). While the tropospheric relative humidity is very sensitive to the large-scale circulation, it is almost invariant with sea surface temperature ([Romps, 2014]). For example, if precipitation increases because sea surface temperature increases without any change in large-scale circulation, then the tropospheric humidity would remain almost constant ([Romps, 2014]), the above-mentioned mechanism cannot take place and there is no amount effect.

\subsection{Discussion}

This study has investigated processes controlling isotopic profiles in idealized conditions. In particular, horizontal gradients in humidity and $\delta D$ were neglected. In reality, these gradients are expected to dampen the humidity and $\delta D$ variations as a function of large-scale vertical velocity ([Risi et al., 2019]).

The large-scale circulation is accounted for through tendencies in humidity, temperature and isotopes that are horizontally homogeneous. This is equivalent to adding an horizontally-constant vertical velocity in each grid point, thus shifting the probability distribution of grid-point vertical velocities $w$. In reality, in case of large-scale ascent, we expect the $w$ distribution to be modified, with a thicker tail corresponding to a larger cloud fraction, while keeping its maximum corresponding to slow radiative subsidence ([Emanuel et al., 1994]). To check this, we would need to analyze LES on large-domains in which a large-scale circulation is allowed to develop, without needing to artificially prescribe large-scale tendencies ([Bretherton and Khairoutdinov, 2015, Khairoutdinov and Emanuel, 2018, 


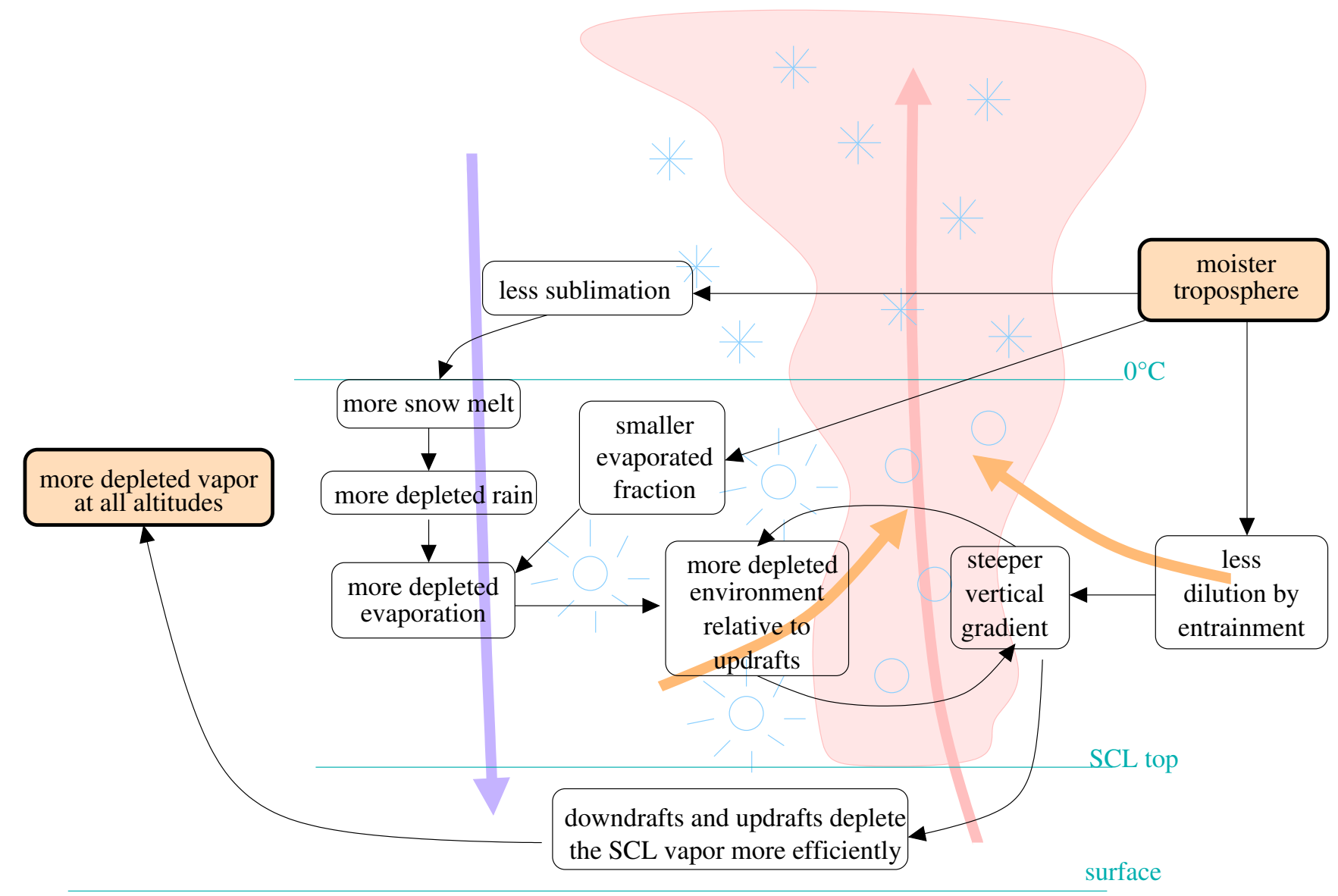

Figure 14: Schematic summarizing how a moister troposphere leads to more depleted vapor in the troposphere. 
Stevens et al., 2019]). According to our analytical model, restricting the large-scale forcing to the cloudy regions would dampen the humidity variations (Text S3) and amplify the $\delta D$ variations as a function of large-scale vertical velocity.

To assess to what what extent our idealized simulations in radiative-convective equilibrium over the ocean are relevant for interpreting observations, it would be useful to compare our LES simulations with different large-scale velocities to in-situ and remote-sensing observations. This raises the question of the spatial scales at which the amount effect can be observed and of the spatial representativeness of both observations and LES simulations. This will also be investigated in a future study.

\subsection{Perspectives}

This paper highlights the important role of snow melt and rain evaporation in depleting the water vapor in case of large-scale ascent. These processes are expected to be even stronger in stratiform regions of meso-scale systems, where all the rain arises from the brutal melting of snow near the melting level, and where the rain evaporation is boosted by the meso-scale downdraft drying the lower troposphere ([Houze, 1977]). This may explain why observations show that stratiform regions are often more depleted than convective regions in squall lines ([Risi et al., 2010, Tremoy et al., 2014]), and why the amount effect is stronger where the fraction of stratiform clouds is larger ([Kurita, 2013, Aggarwal et al., 2016, Sengupta et al., 2020]. To check this hypothesis, we plan to analyze in a future study the dependence of water vapor isotopic profiles to large-scale circulation in LES with different convective organizations, such as squall lines ([Robe and Emanuel, 2001, Muller, 2013]) or tropical cyclones ([Khairoutdinov and Emanuel, 2013, Muller and Romps, 2018]).

Finally, this study highlights the key role of both microphysical processes (evaporation, snow melt) and macrophysical processes (entrainment) in the amount effect. These processes are typically parameterized in LES through the microphysical and sub-grid-scale turbulence schemes in LES. What is the sensitivity of the amount effect to these parameterizations? These processes are even more crudely parameterized in general circulation models (GCMs). How do GCMs represent these processes? More generally, what would be the added value of adding isotopic diagnostics when routinely comparing single-column versions of GCMs to LES simulations? This is yet another question that we plan to address in the future.

\section{Acknowledgements}

This work was granted access to the HPC resources of IDRIS under the allocation 2092 made by GENCI. We thank Giuseppe Torri, Jean-Yves Grandpeix, Sandrine Bony, Nicolas Rochetin, Olivier Pauluis for discussions. C.M. gratefully acknowledges funding from the European Research Council (ERC) under the Euro pean Union's Horizon 2020 research and innovation programme (Project CLUSTER, grant agreement No 805041). The contribution of P.B. was supported by the National Science Foundation under Grant No. AGS-1938108 and by NASA grant NNX13AN47G. Information on SAM can be found on this web page: http://rossby.msrc.sunysb.edu/ $\sim$ marat/SAM.html All simulation outputs used in this article will be submitted to the PANGEA data repository.

\section{References}

[Aggarwal et al., 2016] Aggarwal, P. K., Romatschke, U., Araguas-Araguas, L., Belachew, D., Longstaffe, F. J., Berg, P., Schumacher, C., and Funk, A. (2016). Proportions of convective and stratiform precipitation revealed in water isotope ratios. Nature Geoscience, 9(8):624-629, https://doi.org/10.1038/ngeo2739.

[Bailey et al., 2017] Bailey, A., Blossey, P., Noone, D., Nusbaumer, J., and Wood, R. (2017). Detecting shifts in tropical moisture imbalances with satellite-derived isotope ratios in water vapor. Journal of Geophysical Research: Atmospheres, 122(11):5763-5779.

[Blossey et al., 2010] Blossey, P. N., Kuang, Z., and Romps, D. M. (2010). Isotopic composition of water in the tropical tropopause layer in cloud-resolving simulations of an idealized tropical circulation. J. Geophys. Res., 115:D24309, doi:10.1029/2010JD014554.

[Bony et al., 2008] Bony, S., Risi, C., and Vimeux, F. (2008). Influence of convective processes on the isotopic composition (deltaO18 and deltaD) of precipitation and water vapor in the Tropics. Part 1: Radiative-convective equilibrium and TOGA-COARE simulations. J. Geophys. Res., 113:D19305, doi:10.1029/2008JD009942. 
[Bretherton and Khairoutdinov, 2015] Bretherton, C. S. and Khairoutdinov, M. F. (2015). Convective selfaggregation feedbacks in near-global cloud-resolving simulations of an aquaplanet. Journal of Advances in Modeling Earth Systems, 7(4):1765-1787.

[Chen et al., 2018] Chen, X., Pauluis, O. M., and Zhang, F. (2018). Atmospheric overturning across multiple scales of an mjo event during the cindy/dynamo campaign. Journal of the Atmospheric Sciences, 75(2):381-399.

[Craig and Gordon, 1965] Craig, H. and Gordon, L. I. (1965). Deuterium and oxygen-18 variations in the ocean and marine atmosphere. Stable Isotope in Oceanographic Studies and Paleotemperatures, Laboratorio di Geologia Nucleate, Pisa, Italy:9-130.

[Dansgaard, 1964] Dansgaard (1964). Stable isotopes in precipitation. Tellus, 16:436-468.

[Dauhut et al., 2017] Dauhut, T., Chaboureau, J.-P., Mascart, P., and Pauluis, O. (2017). The atmospheric overturning induced by hector the convector. Journal of the Atmospheric Sciences, 74(10):3271-3284.

[De Rooy et al., 2013] De Rooy, W. C., Bechtold, P., Fröhlich, K., Hohenegger, C., Jonker, H., Mironov, D., Siebesma, A. P., Teixeira, J., and Yano, J.-I. (2013). Entrainment and detrainment in cumulus convection: An overview. Quarterly Journal of the Royal Meteorological Society, 139(670):1-19.

[Dee et al., 2018] Dee, S. G., Nusbaumer, J., Bailey, A., Russell, J. M., Lee, J.-E., Konecky, B., Buenning, N. H., and Noone, D. C. (2018). Tracking the strength of the walker circulation with stable isotopes in water vapor. Journal of Geophysical Research: Atmospheres, 123(14):7254-7270.

[Del Genio and Wu, 2010] Del Genio, A. D. and Wu, J. (2010). The role of entrainment in the diurnal cycle of continental convection. Journal of Climate, 23(10):2722-2738.

[Dessler and Sherwood, 2003] Dessler, A. E. and Sherwood, S. C. (2003). A model of HDO in the tropical tropopause layer. Atmos. Chem. Phys., 3:2173-2181.

[Duan et al., 2018] Duan, S. Q., Wright, J. S., and Romps, D. M. (2018). On the utility (or futility) of using stable water isotopes to constrain the bulk properties of tropical convection. Journal of Advances in Modeling Earth Systems, 10(2):516-529.

[Emanuel et al., 1994] Emanuel, K., Neelin, D., and Bretherton, C. (1994). On large-scale circulations in convecting atmospheres. Quaterly Journal of the Royal Meteorological Society, 120:1111-1143.

[Field et al., 2010] Field, R. D., Jones, D. B. A., and Brown, D. P. (2010). The effects of post-condensation exchange on the isotopic composition of water in the atmosphere. J. Geophy. Res., 115, D24305:doi:10.1029/2010JD014334.

[Field et al., 2014] Field, R. D., Kim, D., LeGrande, A. N., Worden, J., Kelley, M., and Schmidt, G. A. (2014). Evaluating climate model performance in the tropics with retrievals of water isotopic composition from Aura TES. Geophy. Res. Lett., page DOI: 10.1002/2014GL060572.

[Galewsky and Hurley, 2010] Galewsky, J. and Hurley, J. V. (2010). An advection-condensation model for subtropical water vapor isotopic ratios. J. Geophys. Res., 115 (D16):D16115 , doi:10.1029/2009JD013651.

[Galewsky and Rabanus, 2016] Galewsky, J. and Rabanus, D. (2016). A stochastic model for diagnosing subtropical humidity dynamics with stable isotopologues of water vapor. Journal of the Atmospheric Sciences, 73(4):17411753.

[Glenn and Krueger, 2014] Glenn, I. B. and Krueger, S. K. (2014). Downdrafts in the near cloud environment of deep convective updrafts. Journal of Advances in Modeling Earth Systems, 6(1):1-8.

[Godunov, 1959] Godunov, S. K. (1959). Finite-difference methods for the numerical computations of equations of gas dynamics. Math. Sb., 7:271-290.

[Hohenegger and Bretherton, 2011] Hohenegger, C. and Bretherton, C. S. (2011). Simulating deep convection with a shallow convection scheme. Atmospheric Chemistry and Physics, 11:10389-10406.

[Houze, 1977] Houze, R. A. (1977). Structure and dynamics of a tropical squall line system. Mon. Wea. Rev., 105:1540-1567. 
[Johnson et al., 1999] Johnson, R. H., Rickenbach, T. M., Rutledge, S. A., Ciesielski, P. E., and Schubert, W. H. (1999). Trimodal characteristics of tropical convection. Journal of climate, 12(8):2397-2418.

[Khairoutdinov and Emanuel, 2013] Khairoutdinov, M. and Emanuel, K. (2013). Rotating radiative-convective equilibrium simulated by a cloud-resolving model. Journal of Advances in Modeling Earth Systems, 5(4):816825.

[Khairoutdinov and Emanuel, 2018] Khairoutdinov, M. F. and Emanuel, K. (2018). Intraseasonal variability in a cloud-permitting near-global equatorial aquaplanet model. Journal of the Atmospheric Sciences, 75(12):43374355 .

[Khairoutdinov and Randall, 2003] Khairoutdinov, M. F. and Randall, D. A. (2003). Cloud resolving modeling of the arm summer 1997 iop: Model formulation, results, uncertainties, and sensitivities. Journal of the Atmospheric Sciences, 60(4):607-625.

[Kuang and Bretherton, 2006] Kuang, Z. and Bretherton, C. S. (2006). A mass-flux scheme view of a high-resolution simulation of a transition from shallow to deep cumulus convection. Journal of the Atmospheric Sciences, 63(7):1895-1909.

[Kurita, 2013] Kurita, N. (2013). Water isotopic variability in response to mesoscale convective system over the tropical ocean. Journal of Geophysical Research, 118(18):10-376.

[Kurita et al., 2011] Kurita, N., Noone, D., Risi, C., Schmidt, G. A., Yamada, H., , and Yoneyama, K. (2011). Intraseasonal isotopic variation associated with the Madden-Julian Oscillation. J. Geophy. Res., 116:D24, D24101, doi:10.1029/2010JD015209.

[Lacour et al., 2017] Lacour, J.-L., Risi, C., Worden, J., Clerbaux, C., and Coheur, P.-F. (2017). Isotopic signature of convection's depth in water vapor as seen from iasi and tes d observations. Earth Planet. Sci. Lett., 7:9645-9663, doi.org/10.5194/acp-17-9645-2017.

[Lawrence et al., 2004] Lawrence, J. R., Gedzelman, S. D., Dexheimer, D., Cho, H.-K., Carrie, G. D., Gasparini, R., Anderson, C. R., Bowman, K. P., and Biggerstaff, M. I. (2004). Stable isotopic composition of water vapor in the tropics. J. Geophys. Res., 109:D06115, doi:10.1029/2003JD004046.

[Lee and Fung, 2008] Lee, J.-E. and Fung, I. (2008). "Amount effect" of water isotopes and quantitative analysis of post-condensation processes. Hydrological Processes, 22 (1):1-8.

[Lee et al., 2007] Lee, J.-E., Fung, I., DePaolo, D., and Fennig, C. C. (2007). Analysis of the global distribution of water isotopes using the NCAR atmospheric general circulation model. J. Geophys. Res., 112:D16306, doi:10.1029/2006JD007657.

[Lee et al., 2009] Lee, J.-E., Pierrehumbert, R., Swann, A., and Lintner, B. R. (2009). Sensitivity of stable water isotopic values to convective parameterization schemes. Geophy. Res. Lett., 36:3801, doi:10.1029/2009GL040880.

[Merlivat and Jouzel, 1979] Merlivat, L. and Jouzel, J. (1979). Global climatic interpretation of the DeuteriumOxygen 18 relationship for precipitation. J. Geophys. Res., 84:5029-5332.

[Moore et al., 2016] Moore, M., Blossey, P., Muhlbauer, A., and Kuang, Z. (2016). Microphysical controls on the isotopic composition of wintertime orographic precipitation. Journal of Geophysical Research: Atmospheres, 121(12):7235-7253.

[Moore et al., 2014] Moore, M., Kuang, Z., and Blossey, P. N. (2014). A moisture budget perspective of the amount effect. Geophys. Res. Lett., 41:1329-1335, doi:10.1002/2013GL058302.

[Mrowiec et al., 2015] Mrowiec, A. A., Pauluis, O., Fridlind, A., and Ackerman, A. (2015). Properties of a mesoscale convective system in the context of an isentropic analysis. Journal of the Atmospheric Sciences, 72(5):1945-1962.

[Mrowiec et al., 2016] Mrowiec, A. A., Pauluis, O. M., and Zhang, F. (2016). Isentropic analysis of a simulated hurricane. Journal of the Atmospheric Sciences, 73(5):1857-1870.

[Muller, 2013] Muller, C. (2013). Impact of convective organization on the response of tropical precipitation extremes to warming. Journal of climate, 26(14):5028-5043. 
[Muller and Romps, 2018] Muller, C. J. and Romps, D. M. (2018). Acceleration of tropical cyclogenesis by selfaggregation feedbacks. Proceedings of the National Academy of Sciences, page 201719967.

[Pauluis and Mrowiec, 2013] Pauluis, O. M. and Mrowiec, A. A. (2013). Isentropic analysis of convective motions. Journal of the atmospheric sciences, 70(11):3673-3688.

[Randall et al., 2003] Randall, D., Khairoutdinov, M., Arakawa, A., and Grabowski, W. (2003). Breaking the cloud parameterization deadlock. Bulletin of the American Meteorological Society, 84(11):1547-1564.

[Risi et al., 2008] Risi, C., Bony, S., and Vimeux, F. (2008). Influence of convective processes on the isotopic composition (O18 and D) of precipitation and water vapor in the Tropics: Part 2: Physical interpretation of the amount effect. J. Geophys. Res., 113:D19306, doi:10.1029/2008JD009943.

[Risi et al., 2010] Risi, C., Bony, S., Vimeux, F., Chong, M., and Descroix, L. (2010). Evolution of the water stable isotopic composition of the rain sampled along Sahelian squall lines. Quart. J. Roy. Meteor. Soc., 136 (S1):227 -242 .

[Risi et al., 2019] Risi, C., Galewsky, J., Reverdin, G., and Brient, F. (2019). Controls on the water vapor isotopic composition near the surface of tropical oceans and role of boundary layer mixing processes. Atm. Chem. Phys., 19:12235-12260, https://doi.org/10.5194/acp-19-12235-2019.

[Risi et al., 2020] Risi, C., Muller, C., and N, B. P. (2020). What controls the water vapor isotopic composition near the surface of tropical oceans? results from an analytical model constrained by large-eddy simulations. Journal of Advances in Modeling Earth Systems.

[Robe and Emanuel, 2001] Robe, F. R. and Emanuel, K. A. (2001). The effect of vertical wind shear on radiativeconvective equilibrium states. Journal of the atmospheric sciences, 58(11):1427-1445.

[Romps, 2014] Romps, D. M. (2014). An analytical model for tropical relative humidity. Journal of Climate, 27(19):7432-7449.

[Rozanski et al., 1993] Rozanski, K., Araguas-Araguas, L., and Gonfiantini, R. (1993). Isotopic patterns in modern global precipitation. Geophys. Monogr. Seri., AGU, Climate Change in Continental Isotopic records.

[Schmidt et al., 2005] Schmidt, G., Hoffmann, G., Shindell, D., and Hu, Y. (2005). Modelling atmospheric stable water isotopes and the potential for constraining cloud processes and stratosphere-troposphere water exchange. J. Geophys. Res., 110:D21314, doi:10.1029/2005JD005790.

[Sengupta et al., 2020] Sengupta, S., Bhattacharya, S. K., Parekh, A., Nimya, S. S., Yoshimura, K., and Sarkar, A. (2020). Signatures of monsoon intra-seasonal oscillation and stratiform process in rain isotope variability in northern bay of bengal and their simulation by isotope enabled general circulation model. Clim. Dyn, pages https://doi.org/10.1007/s00382-020-05344-w.

[Sherwood, 1996] Sherwood, S. C. (1996). Maintenance of the free tropospheric tropical water vapor distribution. part II: simulation of large-scale advection. J. Clim., 11:2919-2934.

[Stevens and Bony, 2013] Stevens, B. and Bony, S. (2013). What are climate models missing? Science, 340(6136):1053-1054.

[Stevens et al., 2019] Stevens, B., Satoh, M., Auger, L., Biercamp, J., Bretherton, C. S., Chen, X., Düben, P., Judt, F., Khairoutdinov, M., Klocke, D., et al. (2019). Dyamond: The dynamics of the atmospheric general circulation modeled on non-hydrostatic domains. Progress in Earth and Planetary Science, 6(1):61.

[Thayer-Calder and Randall, 2015] Thayer-Calder, K. and Randall, D. (2015). A numerical investigation of boundary layer quasi-equilibrium. Geophysical Research Letters, 42(2):550-556.

[Thompson et al., 2008] Thompson, G., Field, P. R., Rasmussen, R. M., and Hall, W. D. (2008). Explicit forecasts of winter precipitation using an improved bulk microphysics scheme. part ii: Implementation of a new snow parameterization. Monthly Weather Review, 136(12):5095-5115.

[Tremoy et al., 2014] Tremoy, G., Vimeux, F., Soumana, S., Souley, I., Risi, C., Cattani, O., Favreau, G., and Oi, M. (2014). Clustering mesoscale convective systems with laser-based water vapor delta18O monitoring in Niamey (Niger). J. Geophys. Res., 119(9):5079-5103, DOI: 10.1002/2013JD020968. 


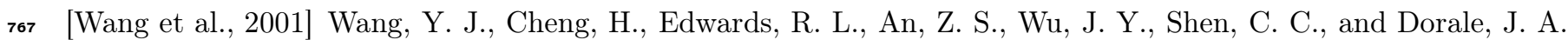
${ }_{768}$ (2001). A high-resolution absolute-dated late Pleistocene Monsoon record from Hulu Cave, China. Science, $769 \quad 294(5550): 2345-8$.

770 [Webb et al., 2015] Webb, M. J., Lock, A. P., Bretherton, C. S., Bony, S., Cole, J. N., Idelkadi, A., Kang, S. M., 771 Koshiro, T., Kawai, H., Ogura, T., et al. (2015). The impact of parametrized convection on cloud feed772 back. Philosophical Transactions of the Royal Society A: Mathematical, Physical and Engineering Sciences, $773 \quad 373(2054): 20140414$.

774 [Worden et al., 2007] Worden, J., Noone, D., and Bowman, K. (2007). Importance of rain evaporation and conti775 nental convection in the tropical water cycle. Nature, 445:528-532. 